\title{
Gluon radiation from a classical point particle. II. Dense gluon fields
}

\author{
K. Kajantie $\oplus^{1, *}$ Larry D. McLerran, ${ }^{2, \dagger}$ and Risto Paatelainen $\oplus^{3,1, *}$ \\ ${ }^{1}$ Helsinki Institute of Physics, University of Helsinki, FI-00014, Helsinki, Finland \\ ${ }^{2}$ Institute for Nuclear Theory, University of Washington, Box 351550, Seattle, Washington 98195, USA \\ ${ }^{3}$ Theoretical Physics Department, CERN, CH-1211 Genève 23, Switzerland
}

(Received 8 January 2020; accepted 18 February 2020; published 11 March 2020)

\begin{abstract}
The goal of this paper is to extend the results of [K. Kajantie, L. D. McLerran, and R. Paatelainen, Gluon radiation from a classical point particle, Phys. Rev. D 100, 054011 (2019)., where formulas were derived for gluonic radiation for a high energy nucleus colliding with a classical colored particle. In [K. Kajantie, L. D. McLerran, and R. Paatelainen, Gluon radiation from a classical point particle, Phys. Rev. D 100, 054011 (2019).], we computed the amplitudes for radiation in the fragmentation region of the particle for a dilute gluonic field. In this paper, we compute the radiation by solving the fluctuation equations of the dense background field in a specific gauge, which makes it simple to solve the asymptotic radiation from an initial condition immediately after the passage of the nucleus. We identify and compute two components of gluon radiation, a bulk component which extends to the central region and bremsstrahlung, which may give rise to an experimentally observable intensity peak in the target fragmentation region.
\end{abstract}

DOI: 10.1103/PhysRevD.101.054012

\section{INTRODUCTION}

In our first paper, Ref. [1], we considered a particle being scattered by a high energy nucleus which was treated as a sheet of colored glass [2,3]. The particle (which we call a quark) being scattered is treated in an approximation where its color field is very weak compared to that of the high energy nucleus, in analogy with the treatment of Refs. [4,5]. In these papers, and many others (see e.g., Refs. [6-19]), gluonic radiation in the central region of the scattering is computed. In this paper, we compute for the first time the distribution of gluonic radiation emitted by this scattering process in the fragmentation region of the target quark. The scattering process is represented by a classical current containing a static target quark and a scattered quark with given momentum. We identify a component which depends on the nuclear saturation scale $Q_{s}^{A}$ and is independent of the momentum of the scattered quark. However, in this classical current approximation there is another component, bremsstrahlung, which depends on the scattered quark momentum, is independent of nuclear effects and gives wrong large transverse

\footnotetext{
*keijo.kajantie@helsinki.fi

mclerran@me.com

risto.sakari.paatelainen@cern.ch
}

Published by the American Physical Society under the terms of the Creative Commons Attribution 4.0 International license. Further distribution of this work must maintain attribution to the author(s) and the published article's title, journal citation, and DOI. Funded by SCOAP. momentum $k_{T}$ behavior. This issue arises due to lack of quantum recoil in the classical current so that to give correctly the gluon distribution for bremsstrahlung contribution at larger $k_{T}$ the model should be improved. The most interesting region of computation, however, is when the $k_{T} \ll Q_{s}^{A}$. In this region, the majority of particles are produced and our computation within a classical current approximation should give a valid description of particle production in the fragmentation region of the target quark.

Investigating this problem is interesting since it solves an old problem: the initial conditions for matter produced in the fragmentation region of high energy scattering [20,21]. In early computations, it was found that the matter produced in the fragmentation region is baryon rich. In the collision the baryons are compressed and there is gluon radiation produced that heats up the system. These early arguments provided a justification for the assertion that one produced a quark gluon plasma in heavy ion collisions. One purpose of this paper is to update these early computations in the modern context of the theory of the color glass condensate (CGC) [22,23].

In later work, we intend to use these initial conditions to provide a theoretically based formulation of the early time evolution of the baryon rich glasma produced in such collisions $[6,7,24]$. Perhaps eventually such consideration may be useful for hydrodynamics studies of the fragmentation region of high energy collisions [25], and the insight engendered by such consideration may allow a more solid theoretical treatment of heavy ion collisions at energies below which one makes a baryon-free central region. 
A simple physical interpretation of what we do is as follows. We first study the process (see Fig. 1)

$$
A+q\left(p_{\text {in }}\right) \rightarrow X+g(k)
$$

in the target fragmentation region of the initial quark so that initial four-momentum ${ }^{1}$ is $p_{\text {in }}=\left(\frac{m}{\sqrt{2}}, \frac{m}{\sqrt{2}}, \mathbf{0}\right)$. The strongly contracted nucleus $A$ is moving along the light cone (LC) $x^{-}=0$ and its large color field [2,3] interacts with the color electric field of the quark. We compute the distribution $d N /\left(d y d^{2} k\right)$ of produced gluons with momentum $k=\left(k^{+}, k^{-}, \mathbf{k}\right), \quad k^{ \pm}=\frac{k_{T}}{\sqrt{2}} e^{ \pm y}, \quad k^{2}=-2 k^{+} k^{-}+k_{T}^{2}=0$. Interactions of the nuclear sheet with this field are shown (see III A) to be the main source of gluon production in the fragmentation region of the static quark. To do this computation, we need a dense nuclear background field $A^{\mu}$ and how it interacts with a small fluctuation field $a^{\mu}$, which gives rise to the electric field of the initial static quark. This interaction follows from Yang-Mills fluctuation equations. The produced gluon distribution is then computed by finding $a^{\mu}$ at asymptotic infinity. We call this the bulk distribution and it is the main quantitative contribution to the goal of this work.

A crucial technical detail of this work is that the nuclear background gauge is chosen so that it only populates the region of space $x^{-}<0$ before the nucleus. Usually it is chosen so that it is excited after the passage of the nucleus. With this choice the fluctuation field for $x^{-}>0$ is a free field and solving it simply requires computing the initial condition at $x^{-}=0^{+}$. Much of the later work is devoted to finding this initial condition.

In addition to the gluons the final state also contains the scattered quark of momentum $p=\left(p^{+}, p^{-}, \mathbf{p}\right), p^{ \pm}=$ $\frac{m_{T}}{\sqrt{2}} e^{ \pm y_{p}}, p^{2}=-2 p^{+} p^{-}+p_{T}^{2}=-m^{2}$ and we also consider more differentially the process

$$
A+q\left(p_{\text {in }}\right) \rightarrow X+q(p)+g(k)
$$

assuming $p$ is a given constant momentum. The process then is gluon production by a classical quark current. We derive the fluctuation equations for this extended case and show that it naturally splits up to the bulk contribution described above and a bremsstrahlung contribution. This bremsstrahlung contribution, however, lacks quark recoil and has to be improved.

Quantum mechanics enters by integrating over an ensemble of classical sources. Diagrammatically, one may describe the setup by saying that the bulk contribution arises by emission of a gluon before the collision, which

\footnotetext{
${ }^{1}$ We use mostly plus light-cone coordinates, $v \equiv v^{\mu}=$ $\left(v^{+}, v^{-}, \mathbf{v}\right),|\mathbf{v}|=v_{T}, v^{ \pm}=-v_{\mp}$ and $v^{i}=v_{i}$. Bold face quantities are two-dimensional transverse vectors, $\mathbf{v}=\left(v_{1}, v_{2}\right)=$ $\left(v_{i}\right), d v_{1} d v_{2}=d^{2} v$. The scalar product of two four-vectors is $\mathbf{x} \cdot \mathbf{y}=-x^{+} y^{-}-x^{-} y^{+}+\mathbf{x} \cdot \mathbf{y}$. The rapidities $y, y_{p}$ always refer to the rest frame of the target particle, $y=y_{\mathrm{CM}}+y_{\text {beam. }}$.
}

then interacts with the nucleus. We show (see II E) that a crucial role for getting a physically valid result is also played by interference with gluons emitted before the collision. Emission of a gluon after the collision, which does not further interact with the nucleus, gives rise to bremsstrahlung. Superficially, the setup is quite different from the case in which both the projectile and target move along opposite light cones. This frame is reached by boosting $y \rightarrow y_{\mathrm{CM}}=y-y_{\text {beam }}$, i.e., by $y \rightarrow \infty$.

The rest of the paper is structured as follows. The classical current is defined and discussed in Sec. II A. The field equations describing the interactions between the current of the initial and accelerated quark and the nuclear background field are described in Sec. II B and solved in Sec. II C. The main result is presented after introducing quantum effects in Sec. II D and discussed in Sec. II E. Bulk and bremsstrahlung contributions and their interference are studied in Secs. III A-III C. Finally, in Sec. III D we show full numerical results for the gluon distribution in the target fragmentation region. Appendixes $\mathrm{A}-\mathrm{C}$ contain details of several subproblems.

\section{TRANSVERSE RADIATION FIELD}

The physics of our problem contains (see Fig. 1) a nucleus moving along the light cone which collides with an initially static quark and accelerates it. The goal is to compute the distribution of gluons produced in this process. In our model of QCD, the nucleus is represented by a strong background color field, the quark by a classical quark current and produced gluons by a transverse radiation field. In this section, we define the quark current, determine equations of motion for a small fluctuation field $a^{\mu}$ and solve them for the radiation field. Part of this material is in paper I; see Ref. [1].

\section{A. The quark current}

Let us first consider the gauge field $A^{\mu}$ induced by the dense projectile nucleus in the longitudinal light-cone gauge $A^{-}=0$, where the gauge field is entirely $A^{+}=$ $A^{+}\left(x^{-}, \mathbf{x}\right)$. The current $J^{\mu}$ for the nucleus corresponds to a Lorentz contracted sheet of infinitesimal thickness at $x^{-}=\epsilon$ with $\epsilon \rightarrow 0$, and it reads

$$
J^{\mu a}\left(x^{-}, \mathbf{x}\right)=\delta^{\mu,+} \delta\left(x^{-}\right) \rho^{a}(\mathbf{x}) .
$$

Here the color charge density on the nuclear sheet is $\rho^{a}(\mathbf{x})$ and the $S U(N)$ adjoint color index $a$ runs from $1, \ldots, N^{2}-1$. The current is independent of $x^{+}$by the extended current conservation law,

$$
D_{\mu} J^{\mu}=\partial_{\mu} J^{\mu}-i g\left[A_{\mu}, J^{\mu}\right]=\partial_{+} J^{+}=0 .
$$

The classical Yang-Mills (CYM) equations with the current defined in Eq. (3) reduce to

$$
D_{\mu} F^{\mu \nu}=J^{\nu} \rightarrow \partial_{T}^{2} A^{+}\left(x^{-}, \mathbf{x}\right)=J^{+}\left(x^{-}, \mathbf{x}\right) .
$$


The dilute target is described by a single color charged particle. The classical current $j^{\mu}$ of the struck particle can be computed by using Wong's equations [26] for the trajectory $x^{\mu}(\tau)$ from the following expression,

$$
j^{\mu}(x)=g \int d \tau Q(\tau) u^{\mu}(\tau) \delta^{(4)}(x-y(\tau)),
$$

where $u^{\mu} \equiv \dot{x}^{\mu}(\tau)$. The particle in a colored field has a classical color charge $Q(\tau) \equiv Q^{a}(\tau) T_{(F)}^{a}$, where $T_{(F)}^{a}$ are the generators in the fundamental representation of $S U(N)$. The classical color charge $Q^{a}$ is assumed to transform as the adjoint representation of the $S U(N)$ gauge group. The average over the space of classical color charges is given by ${ }^{2}$

$$
\left\langle Q^{a} Q^{b}\right\rangle_{Q}=\frac{1}{2} \delta^{a b}, \quad\left\langle Q^{a}\right\rangle_{Q}=0,
$$

where the shorthand notation $\left\langle Q^{a} Q^{b}\right\rangle_{Q}$ is defined as

$$
\left\langle Q^{a} Q^{b}\right\rangle_{Q} \equiv \int d Q Q^{a} Q^{b}, \quad \int d Q=1
$$

and the integration measure $d Q$ over the space of classical color charges is given in Ref. [28].

The covariant conservation $D_{\mu} j^{\mu}=0$ implies that

$$
\frac{d Q^{a}}{d \tau}-i g u^{\mu}\left[A_{\mu}, Q\right]^{a}=0 .
$$

From this equation it follows that the color charge $Q^{a}$ evolves according to

$$
Q^{a} \rightarrow \mathcal{U}_{a b} Q^{b}, \quad \mathcal{U}(x)=\mathcal{P} \exp \left(-i g \int_{x} d y_{\mu} A^{\mu}(y)\right),
$$

where $\mathcal{U}$ is the path ordered adjoint Wilson line.

As shown in Fig. 1, we consider the setup in which a single quark traverses the nucleus with a constant trajectory from the backward light cone with $u^{\mu}=p_{\text {in }}^{\mu} / m$ to the forward light cone with $u^{\mu}=p^{\mu} / \mathrm{m}$. Since the nuclear field extends over the range $x^{-} \in[0, \epsilon]$ with $\epsilon \rightarrow 0$, the current $j^{\mu}$ at $x^{-}<\epsilon$ is modified by color precession in the background field $A^{+}$. This implies that the classical quark current for $x^{-}<\epsilon$ with $\epsilon \rightarrow 0$, expressed in a frame where the quark is at rest with a fixed four-momentum $p_{\text {in }}^{\mu}$, can be written as

$$
\begin{aligned}
j^{\mu a}\left(x^{+}, x^{-}<0, \mathbf{x}\right)= & g \mathcal{U}(\mathbf{0})_{a b} Q^{b} \frac{p_{\mathrm{in}}^{\mu}}{m} \int d \tau \delta\left(x^{-}-u^{-} \tau\right) \\
& \times \delta\left(x^{+}-u^{+} \tau\right) \delta^{(2)}(\mathbf{x}-\mathbf{u} \tau) \\
= & g \mathcal{U}(\mathbf{0})_{a b} Q^{b} \mathcal{J}^{\mu}\left(x^{+}, x^{-}<0, \mathbf{x}\right),
\end{aligned}
$$

\footnotetext{
${ }^{2}$ See also the discussion in Ref. [27].
}

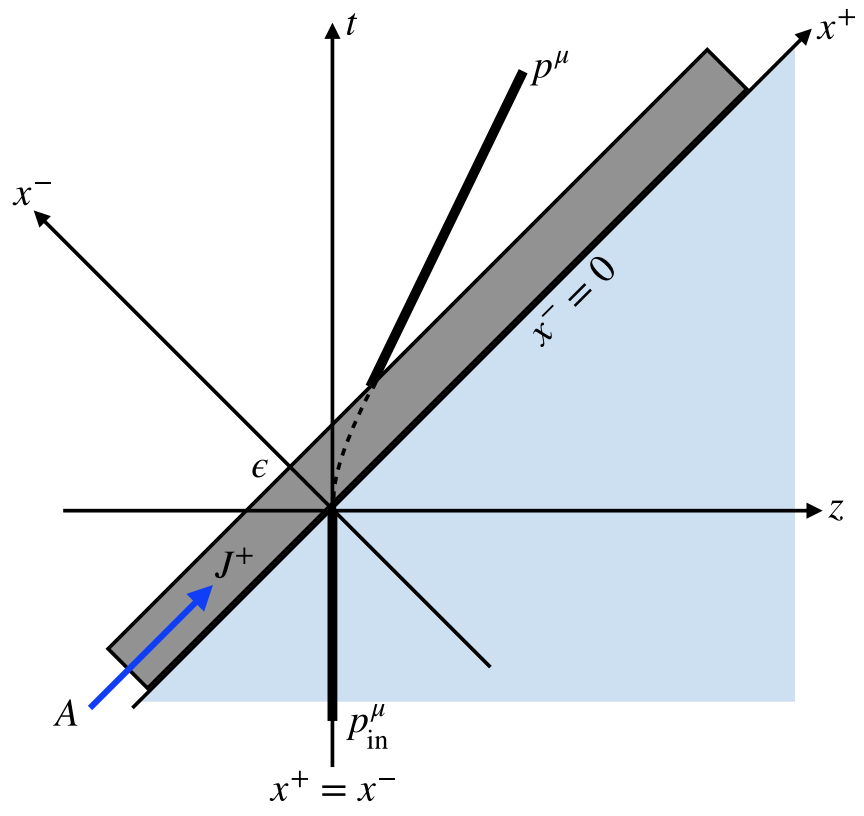

FIG. 1. Nucleus $A$ moving along the light cone $x^{-}=0$ collides with a static quark in the origin, interacts with its color electric field and accelerates it to a constant momentum (thick lines). The nuclear vacuum equivalent background color field in light-cone (LC) gauge $A^{-}=0$ is chosen so that it is nonzero at $x^{-}<0$ before the collision (light blue area) and 0 after it. The asymptotic color fluctuation field, which gives the gluon distribution, can then be simply solved from initial conditions formulated at $x^{-}=\epsilon \rightarrow 0$.

where we have defined

$$
\mathcal{J}^{\mu}\left(x^{+}, x^{-}<0, \mathbf{x}\right) \equiv \frac{p_{\text {in }}^{\mu}}{p_{\text {in }}^{-}} \delta\left(x^{+}-x^{-}\right) \delta^{(2)}(\mathbf{x}) .
$$

Here the adjoint Wilson line $\mathcal{U}$ for $x^{-} \in[0, \epsilon]$ is given by

$\mathcal{U}\left(x^{-}=\epsilon, \mathbf{x}\right)=\mathcal{P} \exp \left(-i g T_{(A)}^{a} \int_{x^{-}}^{\infty} d y^{-} A_{a}^{+}\left(y^{-}, \mathbf{x}\right)\right)$,

and $T_{(A)}^{a}$ are the generators of $S U(N)$ in the adjoint representation. In the limit $\epsilon \rightarrow 0$ this simplifies to

$$
\mathcal{U}(\mathbf{x})=\mathcal{P} \exp \left(-i g T_{(A)}^{a} \Phi_{a}(\mathbf{x})\right)
$$

where the field $\Phi_{a}$ satisfies $\partial_{T}^{2} \Phi_{a}(\mathbf{x})=\rho_{a}(\mathbf{x})$. After the collision at $x^{-}>0$, the quark at rest accelerates to fixed momentum $p^{\mu}$. The corresponding color current reads

$$
j^{\mu a}\left(x^{+}, x^{-}>0, \mathbf{x}\right)=g Q^{a} \mathcal{J}^{\mu}\left(x^{+}, x^{-}>0, \mathbf{x}\right),
$$

where 
$\mathcal{J}^{\mu}\left(x^{+}, x^{-}>0, \mathbf{x}\right) \equiv \frac{p^{\mu}}{p^{-}} \delta\left(x^{+}-\frac{p^{+}}{p^{-}} x^{-}\right) \delta^{(2)}\left(\mathbf{x}-\mathbf{p} \frac{x^{-}}{p^{-}}\right)$

Putting Eqs. (11) and (15) together, we find that the final total quark current reads

$$
\begin{aligned}
j^{\mu a}\left(x^{+}, x^{-}, \mathbf{x}\right)= & g \mathcal{U}(\mathbf{0})_{a b} Q^{b} \frac{p_{\text {in }}^{\mu}}{p_{\text {in }}^{-}} \delta\left(x^{+}-x^{-}\right) \delta^{(2)}(\mathbf{x}) \theta\left(-x^{-}\right) \\
& +g Q^{a} \frac{p^{\mu}}{p^{-}} \delta\left(x^{+}-\frac{p^{+}}{p^{-}} x^{-}\right) \\
& \times \delta^{(2)}\left(\mathbf{x}-\mathbf{p} \frac{x^{-}}{p^{-}}\right) \theta\left(x^{-}\right) .
\end{aligned}
$$

\section{B. Equations of motion}

We are interested in the radiation field produced after the quark-nucleus collision; i.e., knowing the gauge fields for $x^{-}<0$, what are the gauge fields far in the future at $x^{-}>0$ ? The radiation field can be obtained by adding a small perturbation $a^{\mu}$ to the background gauge field $A^{\mu}$ of the nucleus

$$
A^{\mu}=A^{\mu}+a^{\mu} \quad \text { with } \quad J^{\mu}=J^{\mu}+j^{\mu},
$$

and solving the corresponding CYM equations to linear order in the small fluctuation field $a^{\mu}$. However, in the initial longitudinal light-cone gauge where the gauge field is entirely $A^{+}$, the gauge field is singular at $x^{-}=0$. In order to avoid this unpleasant feature, it is optimal to choose a gauge where the gauge field $A^{\mu}$ is a two-dimensional transverse field $A^{i}$ for $x^{-}<0$ so that it vanishes for $x^{-}>$ 0 before the passage of the nucleus. This goal can be achieved by choosing suitably the gauge rotation that connects the longitudinal $A^{+}$gauge to the transverse $A^{i}$ gauge. Following the discussion in [1], this amounts to solving the equation

$$
\partial_{-} \mathcal{U}^{\dagger}\left(x^{-}, \mathbf{x}\right)=-i g A^{+}\left(x^{-}, \mathbf{x}\right) \mathcal{U}^{\dagger}\left(x^{-}, \mathbf{x}\right),
$$

where $\mathcal{U}$ is the gauge rotation matrix transforming $A^{+}$to 0 . A solution where $\mathcal{U}$ is one for $x^{-}>0$ is given by the adjoint Wilson line in (13). For $x^{-}<0$, the two-dimensional gauge field associated with this rotation is

$$
-i g A^{i}(\mathbf{x})=\mathcal{U}(\mathbf{x}) \partial^{i} \mathcal{U}^{\dagger}(\mathbf{x})
$$

In this new gauge (hereby referred as the transverse LC gauge), the equation of motion for the transverse fluctuation field $a^{i}$ can be written as $^{3}$ [1]

\footnotetext{
${ }^{3}$ This is obtained from the general fluctuation equation $D^{2} a^{\mu}-D^{\mu}(D \cdot a)-2 i g F^{\mu \nu} a_{\nu}=\tilde{j}^{\mu}$.
}

$$
D^{2} a^{i}=\left(-2 \partial^{+} \partial^{-}+D_{T}^{2}\right) a^{i}=\tilde{j}^{i}-D^{i} \frac{1}{\partial^{-}} \tilde{j}^{-},
$$

where the notation $D_{T}^{2} \equiv D_{k} D_{k}$ is introduced. The current $\tilde{j}^{\mu}$ is related to the current $j^{\mu}$ in the $A^{+}$gauge via the transformation $\tilde{j}^{\mu} \rightarrow \mathcal{U} j^{\mu}$. Using the relations

$$
\begin{aligned}
& D_{k} S=\mathcal{U} \partial_{k}\left(\mathcal{U}^{\dagger} S\right), \\
& D_{T}^{2} S=\mathcal{U} \partial_{T}^{2}\left(\mathcal{U}^{\dagger} S\right),
\end{aligned}
$$

and noting that $\left[\partial^{ \pm}, \mathcal{U}\right]=0$ yields the expression

$$
\square\left(\mathcal{U}^{\dagger} a^{i}\right)=j^{i}-\partial^{i} \frac{1}{\partial^{-}} j^{-} \equiv j_{\mathrm{eff}}^{i},
$$

where $\square \equiv-2 \partial^{+} \partial^{-}+\partial_{T}^{2}$ is the d'Alembert operator.

\section{Solving the radiation field}

We now apply the special gauge choice made for the background field, i.e., vanishing $A^{i}$ at $x^{-}>0$. For $x^{-}>0$ the gauge field then is a free field and can simply be solved at infinity by giving the field at $x^{-}=0$. In [1] it was proven that the field $a_{i}$ is continuous at $x^{-}=0$.

For $x^{-}<0$, the full solution of Eq. (23) can be written down in terms of the retarded Green function,

$$
\begin{aligned}
a^{i}\left(x^{+}, x^{-}<0, \mathbf{x}\right)= & a_{\mathrm{in}}^{i}+\mathcal{U}(\mathbf{x}) \int d^{4} y G_{R}(x-y) \\
& \times j_{\mathrm{eff}}^{i}\left(y^{+}, y^{-}<0, \mathbf{y}\right)
\end{aligned}
$$

where the "incoming" field $a_{\text {in }}^{i}$ is the solution of the homogeneous fluctuation equation for $x^{-}<0$ with no sources. Note that the incoming field vanishes since the quark is initially at rest. Now, in order to obtain the radiation field, we have to find the transverse gauge field after the nucleus has passed at $x^{-}>0$. The corresponding fluctuation equation is easily obtained by noting that $\mathcal{U}=1$ in Eq. (23). This yields

$$
\square a^{i}=j_{\mathrm{eff}}^{i}\left(x^{+}, x^{-}>0, \mathbf{x}\right) .
$$

The general solution in terms of the advanced Green function reads

$$
\begin{aligned}
a^{i}\left(x^{+}, x^{-}>0, \mathbf{x}\right)= & a_{\mathrm{out}}^{i}+\int d^{4} y G_{A}(x-y) \\
& \times j_{\mathrm{eff}}^{i}\left(y^{+}, y^{-}>0, \mathbf{y}\right),
\end{aligned}
$$

where the outgoing field $a_{\text {out }}^{i}$ is the solution of the homogenous equation $\square a^{i}\left(x^{-}>0\right)=0$.

The solution of the transverse radiation field at the surface $x^{-}=0$ is then obtained by taking the difference between the outgoing and incoming fields, 


$$
\begin{aligned}
& a_{\mathrm{rad}}^{i}\left(x^{+}, x^{-}=0, \mathbf{x}\right) \\
& =\mathcal{U}(\mathbf{x}) \int d^{4} y G_{R}(x-y) j_{\mathrm{eff}}^{i}\left(y^{+}, y^{-}<0, \mathbf{y}\right) \\
& \quad-\int d^{4} y G_{A}(x-y) j_{\mathrm{eff}}^{i}\left(y^{+}, y^{-}>0, \mathbf{y}\right) .
\end{aligned}
$$

Note that in taking the difference the left-hand sides of Eqs. (24) and (26) cancel since both are evaluated at $x^{-}=0$ and $a_{i}$ is continuous there. Using the notation introduced in Eqs. (11) and (15), the radiation field at $x^{-}=0$ with all color indices can be written as

$$
\begin{aligned}
& a_{\mathrm{rad}}^{i a}\left(x^{+}, x^{-}=0, \mathbf{x}\right) \\
& =g \mathcal{U}_{a d}(\mathbf{x}) \mathcal{U}_{d b}(\mathbf{0}) Q^{b} \int d^{4} y G_{R}(x-y) \mathcal{J}_{\mathrm{eff}}^{i}\left(y^{+}, y^{-}<0, \mathbf{y}\right) \\
& \quad-g Q^{a} \int d^{4} y G_{A}(x-y) \mathcal{J}_{\mathrm{eff}}^{i}\left(y^{+}, y^{-}>0, \mathbf{y}\right)
\end{aligned}
$$

where the source term

$$
\mathcal{J}_{\text {eff }}^{i}(y) \equiv \mathcal{J}^{i}(y)-\partial^{i} \frac{1}{\partial^{-}} \mathcal{J}^{-}(y)
$$

and $\mathcal{J}^{\mu}$ for $y^{-}<0$ and $y^{-}>0$ is defined in Eqs. (12) and (16), respectively.

From Eq. (28) we observe that the gluon radiation comes from two different contributions: The first contribution on the right-hand side in Eq. (28) is unique to QCD, and arises from the disturbance of a Coulomb field composed of colored gluons that is disturbed during the collision process $q A \rightarrow g$. The second contribution represents the classical electrodynamics (ED)-like gluon radiation caused by the acceleration of the initial static quark by the nuclear sheet to an unspecied transverse momentum.

What we are then interested in is the radiation field in momentum space. This is given by the Fourier transform

$$
a_{\mathrm{rad}}^{i}\left(k^{+}, k^{-}, \mathbf{k}\right)=\int d^{4} x e^{-i k \cdot x} a_{\mathrm{rad}}^{i}\left(x^{+}, x^{-}, \mathbf{x}\right) .
$$

To construct the full radiation field in momentum space we first note that the radiation field is a free field at $x^{-}>0$. From this one can conclude that

$$
\begin{aligned}
a_{\mathrm{rad}}^{i}\left(k^{-}, k^{+}, \mathbf{k}\right) & =\frac{a_{\mathrm{rad}}^{i}\left(k^{-}, x^{-}=0, \mathbf{k}\right)}{i\left(k^{+}-\frac{k_{T}^{2}}{2 k^{-}}-i \epsilon\right)} \\
& =\frac{-2 i k^{-}}{k^{2}+i \epsilon} a_{\mathrm{rad}}^{i}\left(k^{-}, \mathbf{k}\right),
\end{aligned}
$$

where $k^{-} a_{\mathrm{rad}}^{i}\left(k^{-}, x^{-}=0, \mathbf{k}\right)$ is the Fourier transform of the boundary value in Eq. (28). Using $k^{-} \rightarrow i \partial_{+}$and substituting Eq. (31) into Eq. (30) yields the result $i k^{2} a_{\mathrm{rad}}^{i}(k)=\int d x^{+} d^{2} x e^{i\left(k^{-} x^{+}-\mathbf{k} \cdot \mathbf{x}\right)} 2 i \partial_{+} a_{\mathrm{rad}}^{i}\left(x^{+}, x^{-}=0, \mathbf{x}\right)$.

Introducing the following notation in Eq. (28),

$$
\begin{aligned}
& a_{\mathrm{ini}}^{i}\left(x^{+}, x^{-}=0, \mathbf{x}\right) \equiv \int d^{4} y G_{R}(x-y) \mathcal{J}_{\mathrm{eff}}^{i}\left(y^{+}, y^{-}<0, \mathbf{y}\right), \\
& a_{\mathrm{fin}}^{i}\left(x^{+}, x^{-}=0, \mathbf{x}\right) \equiv \int d^{4} y G_{A}(x-y) \mathcal{J}_{\mathrm{eff}}^{i}\left(y^{+}, y^{-}>0, \mathbf{y}\right),
\end{aligned}
$$

considerably simplifies the expression in Eq. (32), yielding the compact expression

$$
\begin{aligned}
i k^{2} a_{\mathrm{rad}}^{i a}\left(k^{+}, k^{-}, \mathbf{k}\right)= & g \mathcal{U}_{d b}(\mathbf{0}) Q^{b} \int d^{2} x e^{-i \mathbf{k} \cdot \mathbf{x}} \mathcal{U}_{a d}(\mathbf{x}) v^{i}(\mathbf{x}) \\
& -g Q^{a} \int d^{2} x e^{-i \mathbf{k} \cdot \mathbf{x}} u^{i}(\mathbf{x}),
\end{aligned}
$$

where the fields $v^{i}$ and $u^{i}$ are defined as

$$
\begin{aligned}
& v^{i}(\mathbf{x}) \equiv 2 i \int d x^{+} e^{i k^{-} x^{+}} \partial_{+} a_{\mathrm{ini}}^{i}\left(x^{+}, x^{-}=0, \mathbf{x}\right), \\
& u^{i}(\mathbf{x}) \equiv 2 i \int d x^{+} e^{i k^{-} x^{+}} \partial_{+} a_{\mathrm{fin}}^{i}\left(x^{+}, x^{-}=0, \mathbf{x}\right) .
\end{aligned}
$$

Here $v_{i}$ is sourced by the initial static part of the quark current; likewise $u_{i}$ is sourced by the recoil quark. Physics of gluon distribution is obtained from the square of Eq. (34) and it comes in two parts: The first term describes gluons arising from the interaction of the nuclear sheet with the Coulomb field of the initial quark, the second emission from the kicked quark. Interference effects are important and are discussed in the next subsection; see Eq. (43).

The Green's function integrals in Eq. (33) are carried out in detail in Appendix A and lead to the results

$$
\begin{aligned}
& v^{i}(\mathbf{x})=-\frac{i}{2 \pi} \frac{x^{i}}{x_{T}} 2 \sqrt{2} k^{-} K_{1}\left(\sqrt{2} k^{-} x_{T}\right)=-i \sqrt{2} E_{i}\left(k^{-}, \mathbf{x}\right), \\
& u^{i}(\mathbf{x})=-\frac{i}{2 \pi} e^{i \frac{k^{-}}{p} \mathbf{p} \cdot \mathbf{x}} \frac{x^{i}}{x_{T}} \frac{2 m k^{-}}{p^{-}} K_{1}\left(\frac{m k^{-}}{p^{-}} x_{T}\right)
\end{aligned}
$$

where $K_{1}$ is the modified Bessel function of the second kind. Note that the field $v^{i} \sim \partial_{+} a_{\text {ini }}^{i}$ corresponds to the color electric field of a static quark in vacuum, where the transverse electric field $E_{i}$ along $x^{-}=0$ is generated by the Coulombic vector potential (in $a^{-}=0$ gauge) [1],

$$
\begin{aligned}
F_{+i} & =\partial_{+} a_{\mathrm{ini}}^{i}\left(x^{+}, 0, \mathbf{x}\right) \equiv-\frac{1}{\sqrt{2}} E^{i}\left(x^{+}, 0, \mathbf{x}\right) \\
& =-\frac{x^{i}}{4 \pi \sqrt{2}\left[\left(x^{+}\right)^{2} / 2+x_{T}^{2}\right]^{3 / 2}}
\end{aligned}
$$


with the Fourier transform

$$
E_{i}\left(k^{-}, \mathbf{x}\right)=\int d x^{+} e^{i k^{-} x^{+}} E_{i}\left(x^{+}, 0, \mathbf{x}\right)=\frac{x_{i}}{x_{T}} \frac{k^{-}}{\pi} K_{1}\left(\sqrt{2} k^{-} x_{T}\right) .
$$

For the field $u^{i} \sim \partial_{+} a_{\text {fin }}^{i}$ there would also be a magnetic field.

\section{Introducing quantum effects}

The number spectrum of produced gluons is obtained by considering the relation

$$
16 \pi^{3} \frac{d N}{d y d^{2} k}=\frac{1}{N} \sum_{\lambda}\left\langle\left|\mathcal{M}_{\lambda}(k)\right|^{2}\right\rangle_{Q, \rho},
$$

where the gluon four-momentum $k$ is parametrized in terms of momentum space rapidity $y$ and the subscript $\langle\ldots\rangle_{\rho}$ denotes an average over the set of color sources. The classical gluon production amplitude ${ }^{4}$ is given by

$$
\mathcal{M}_{\lambda}(k)=k^{2} a_{\mathrm{rad}}^{i}(k) \varepsilon_{\lambda, i},
$$

where the sum over the gluon transverse polarization vectors is

$$
\sum_{\lambda} \varepsilon_{\lambda, i} \varepsilon_{\lambda, j}^{*}=\delta_{i j}
$$

By substituting Eq. (34) into Eq. (39), we obtain

$$
\begin{aligned}
16 \pi^{3} \frac{d N}{d y d^{2} k}= & \frac{g^{2}}{N}\langle| \mathcal{U}_{d b}(\mathbf{0}) Q^{b} \int d^{2} x e^{-i \mathbf{k} \cdot \mathbf{x}} \mathcal{U}_{a d}(\mathbf{x}) v^{i}(\mathbf{x}) \\
& \left.-\left.Q^{a} \int d^{2} x e^{-i \mathbf{k} \cdot \mathbf{x}} u^{i}(\mathbf{x})\right|^{2}\right\rangle_{Q, \rho}
\end{aligned}
$$

Following the straightforward calculation of the color algebra given in Appendix $\mathrm{C}$ yields the result

$$
\begin{aligned}
16 \pi^{3} \frac{d N}{d y d^{2} k}= & g^{2} C_{F} \int d^{2} x d^{2} y e^{-i \mathbf{k} \cdot(\mathbf{x}-\mathbf{y})} \\
& \times\left[S(\mathbf{x}-\mathbf{y}) v^{i}(\mathbf{x}) v^{i *}(\mathbf{y})-S(\mathbf{x}) v^{i}(\mathbf{x}) u^{i *}(\mathbf{y})\right. \\
& \left.-S(\mathbf{y}) u^{i}(\mathbf{x}) v^{i *}(\mathbf{y})+u^{i}(\mathbf{x}) u^{i *}(\mathbf{y})\right]
\end{aligned}
$$

where the color factor $C_{F}=\left(N^{2}-1\right) / 2 N$ and the scattering information between the projectile nucleus and the target quark are encoded in the two-point correlator of two Wilson lines in the adjoint representation

$$
\begin{aligned}
S(\mathbf{x}-\mathbf{y}) & \equiv \frac{1}{N^{2}-1}\left\langle\operatorname{Tr}\left(\mathcal{U}(\mathbf{x}) \mathcal{U}^{\dagger}(\mathbf{y})\right)\right\rangle_{\rho} \\
S(\mathbf{x}) & \equiv \frac{1}{N^{2}-1}\left\langle\operatorname{Tr}\left(\mathcal{U}(\mathbf{x}) \mathcal{U}^{\dagger}(\mathbf{0})\right)\right\rangle_{\rho}, \\
S(\mathbf{y}) & \equiv \frac{1}{N^{2}-1}\left\langle\operatorname{Tr}\left(\mathcal{U}(\mathbf{y}) \mathcal{U}^{\dagger}(\mathbf{0})\right)\right\rangle_{\rho}
\end{aligned}
$$

The explicit calculation of Wilson line correlators in Eq. (44) within the approximation of the McLerranVenugopalan (MV) model [29], where the fluctuations in the nuclear color field sources are Gaussian, is given in Appendix B. This yields the result

$$
S(\mathbf{x}-\mathbf{y})=\exp \left[-\left(Q_{s}^{A}\right)^{2} D(\mathbf{x}-\mathbf{y})\right],
$$

where the dipole function $D$ is given by

$$
D(\mathbf{x}-\mathbf{y}) \equiv \int \frac{d^{2} \mathbf{p}}{(2 \pi)^{2}} \frac{2}{\left(\mathbf{p}^{2}+m_{\mathrm{IR}}^{2}\right)^{2}}\left(1-e^{i \mathbf{p} \cdot(\mathbf{x}-\mathbf{y})}\right)
$$

and $m_{\mathrm{IR}}$ is an infrared regulator.

Let us then Fourier transform the expression obtained in Eq. (43) to momentum space via convolution in twodimensional momentum space defined as

$$
\mathcal{F}[f \otimes g] \equiv \int \frac{d^{2} h}{(2 \pi)^{2}} f(\mathbf{h}-\mathbf{k}) g(\mathbf{h})
$$

First, consider the term with $S(\mathbf{x}-\mathbf{y})$ in Eq. (43). By introducing the following change of variables, $\mathbf{x}=\mathbf{b}+\frac{1}{2} \mathbf{r}$ and $\mathbf{y}=\mathbf{b}-\frac{1}{2} \mathbf{r}$, we obtain

$$
\int d^{2} r S(\mathbf{r}) e^{-i \mathbf{k} \cdot \mathbf{r}} \int d^{2} b v^{i}\left(\mathbf{b}+\frac{1}{2} \mathbf{r}\right) v^{i *}\left(\mathbf{b}-\frac{1}{2} \mathbf{r}\right) .
$$

Since $S$ only depends on $\mathbf{r}$ the $\mathbf{b}$-integration can be brought to the $v^{i} v^{i *}$ terms and carried out with two different Fourier momenta. This yields the result

$$
\int \frac{d^{2} q}{(2 \pi)^{2}} S(\mathbf{k}-\mathbf{h}) v^{i}(\mathbf{h}) v^{i *}(\mathbf{h})
$$

where the Fourier transforms of $v^{i}$ and $u^{i}$ are given in Appendix A. The second and the third term in Eq. (43) follow similarly by considering the Fourier transforms of $S, v$ and $u$. This yields the result

$$
\begin{gathered}
\int d^{2} x e^{-i \mathbf{k} \cdot \mathbf{x}} S(\mathbf{x}) v^{i}(\mathbf{x}) \int d^{2} y e^{i \mathbf{k} \cdot \mathbf{y}} u^{i *}(\mathbf{y}) \\
=\int \frac{d^{2} h}{(2 \pi)^{2}} S(\mathbf{k}-\mathbf{h}) v^{i}(\mathbf{h}) u^{i *}(\mathbf{k}),
\end{gathered}
$$

\footnotetext{
${ }^{4}$ Note that we work in the transverse LC gauge where $a^{-}=0$.
} 


$$
\begin{gathered}
\int d^{2} x e^{-i \mathbf{k} \cdot \mathbf{x}} u^{i}(\mathbf{x}) \int d^{2} y e^{i \mathbf{k} \cdot \mathbf{y}} S(\mathbf{y}) v^{i *}(\mathbf{y}) \\
=\int \frac{d^{2} h}{(2 \pi)^{2}} S(\mathbf{k}-\mathbf{h}) v^{i *}(\mathbf{h}) u^{i}(\mathbf{k})
\end{gathered}
$$

Finally, the last term is directly a product $u^{i}(\mathbf{k}) u^{i *}(\mathbf{k})$ of Fourier transforms, but using the normalization condition

$$
\int \frac{d^{2} h}{(2 \pi)^{2}} S(\mathbf{h})=1
$$

it can be combined with the three other terms and one can write the outcome in the form

$$
\begin{aligned}
16 \pi^{3} \frac{d N}{d y d^{2} k}= & g^{2} C_{F} \int \frac{d^{2} h}{(2 \pi)^{2}} S(\mathbf{k}-\mathbf{h})\left(v^{i}(\mathbf{h})-u^{i}(\mathbf{k})\right) \\
& \times\left(v^{* i}(\mathbf{h})-u^{* i}(\mathbf{k})\right) .
\end{aligned}
$$

By substituting the explicit expression for $v$ and $u$ [see Appendix A Eqs. (A13) and (A19)], we find a very compact and elegant expression for the gluon radiation spectrum,

$$
\begin{aligned}
\left(\frac{16 \pi^{3}}{g^{2} C_{F}}\right) \frac{d N}{d y d^{2} k}= & 2 \int \frac{d^{2} h}{(2 \pi)^{2}} S(\mathbf{k}-\mathbf{h}) \\
& \times\left[\frac{h^{i}}{h_{T}^{2}+2\left(k^{-}\right)^{2}}-\frac{k^{i}-\xi p^{i}}{|\mathbf{k}-\xi \mathbf{p}|^{2}+\xi^{2} m^{2}}\right]^{2},
\end{aligned}
$$

where we have defined $\xi \equiv k^{-} / p^{-}$. This is the main result of our work.

\section{E. Discussion of the main result in Eq. (53)}

\section{Relation to known results}

As a first check one can compare with known results in the central region, which corresponds to $y \rightarrow \infty$ in Eq. (53). In this limit, $k^{-} \sim e^{-y} \rightarrow 0, \xi=k^{-} / p^{-} \rightarrow 0$, and the bracketed factor becomes

$$
\left(\frac{h^{i}}{h_{T}^{2}}-\frac{k^{i}}{k_{T}^{2}}\right)^{2}=\frac{|\mathbf{k}-\mathbf{h}|^{2}}{h_{T}^{2} k_{T}^{2}} .
$$

This shows immediately the relation to Eq. (23) of Ref. [12], where the gluon production amplitude is given for $p+A$ collisions in a similar temporal LC gauge. The rhs of Eq. (53) thus becomes

$$
\left.\left(\frac{16 \pi^{3}}{g^{2} C_{F}}\right) \frac{d N}{d y d^{2} k}\right|_{\text {bulk }, y \rightarrow \infty}=2 \int \frac{d^{2} h}{(2 \pi)^{2}} S(\mathbf{k}-\mathbf{h}) \frac{|\mathbf{k}-\mathbf{h}|^{2}}{h_{T}^{2} k_{T}^{2}},
$$

reminiscent of known results for gluon production in $p+A$ in Ref. [10] (the color correlator on the proton side is missing). More specifically, using for $S$ the standard large momentum approximation $k_{T} \gg Q_{s}^{A}$ [see the derivation in Eq. (B19) in Appendix B],

$$
S(\mathbf{k})=\frac{2 Q_{s}^{2}}{k_{T}^{4}}+\mathcal{O}\left(\frac{Q_{s}^{4}}{k_{T}^{6}}\right)
$$

where the nonlinearities of the projectile color field are unimportant, the rhs in Eq. (55) becomes a GunionBertsch-type [30] infrared divergent spectrum in the central region

$$
\left.\left(\frac{16 \pi^{3}}{g^{2} C_{F}}\right) \frac{d N}{d y d^{2} k}\right|_{\text {bulk }} \approx \frac{4\left(Q_{s}^{A}\right)^{2}}{k_{T}^{2}} \int \frac{d^{2} h}{(2 \pi)^{2}} \frac{1}{|\mathbf{k}-\mathbf{h}|^{2} h_{T}^{2}} .
$$

\section{Separation of bulk and bremsstrahlung}

To interpret the structure of the result it turns out to be useful to add and subtract

$$
\frac{1}{\sqrt{2}} i E\left(k^{-}, \mathbf{k}\right)=\frac{k^{i}}{k_{T}^{2}+2\left(k^{-}\right)^{2}}
$$

inside the brackets in Eq. (53). This yields the result

$$
\left(\frac{16 \pi^{3}}{g^{2} C_{F}}\right) \frac{d N}{d y d^{2} k}=2 \int \frac{d^{2} h}{(2 \pi)^{2}} S(\mathbf{k}-\mathbf{h})\left[\mathcal{M}_{\text {bulk }}^{i}+\mathcal{M}_{\text {brems }}^{i}\right]^{2},
$$

where we have introduced the compact notation

$$
\mathcal{M}_{\text {bulk }}^{i} \equiv \frac{h^{i}}{h_{T}^{2}+2\left(k^{-}\right)^{2}}-\frac{k^{i}}{k_{T}^{2}+2\left(k^{-}\right)^{2}},
$$

and

$$
\mathcal{M}_{\text {brems }}^{i} \equiv \frac{k^{i}}{k_{T}^{2}+2\left(k^{-}\right)^{2}}-\frac{k^{i}-\xi p^{i}}{|\mathbf{k}-\xi \mathbf{p}|^{2}+\xi^{2} m^{2}} .
$$

What we have hereby gained is a separation of scales. The bulk term, denoted as $\mathcal{M}_{\text {bulk }}^{i}$, now depends on the convolution and the produced gluon momenta; there is no dependence on the kicked quark momentum. The bremsstrahlung term, denoted as $\mathcal{M}_{\text {brems }}^{i}$, on the other hand, is completely independent of the convolution momentum so that, due to the normalization of $S(q)$ in Eq. (52), the integration over $h^{i}$ can be carried out and $S(q)$ and all nuclear effects are entirely eliminated. Since $\xi=k^{-} / p^{-}$ and $k^{-} \sim e^{-y}$ it also manifestly vanishes in the central region where $y$ is large.

In the following section, we address the three separate contributions to the gluon radiation spectrum obtained in Eq. (59). The first comes from $\left|\mathcal{M}_{\text {bulk }}^{i}\right|^{2}$, and it is the contribution from the interaction between the nuclear sheet and the electric field of the static initial quark. The second is the bremsstrahlung contribution coming from $\left|\mathcal{M}_{\text {brems }}^{i}\right|^{2}$. 
Taken literally as it stands in Eq. (61) it contains no dependence on the nucleus. It gives precisely classical ED gluon emission distribution going $\sim 1 / k_{T}^{2}$ even at asymptotic $k_{T}$. We show that this is due to the assumed constant kick and has to be corrected by a momentum $q$ absorbed from the nucleus. Finally, the third contribution comes from the possible interference contribution between the bulk and bremsstrahlung.

\section{PROPERTIES AND NUMERICS OF BULK AND BREMSSTRAHLUNG}

\section{A. Bulk contribution}

Let us first consider the bulk contribution in Eq. (59) which, after opening the brackets, yields the expression

$$
\begin{aligned}
\left.\left(\frac{16 \pi^{3}}{g^{2} C_{F}}\right) \frac{d N}{d y d^{2} k}\right|_{\text {bulk }} \equiv & 2 \int \frac{d^{2} h}{(2 \pi)^{2}} S(\mathbf{k}-\mathbf{h})\left[\frac{h_{T}^{2}}{\left(h_{T}^{2}+2\left(k^{-}\right)^{2}\right)^{2}}\right. \\
& -\frac{2 \mathbf{h} \cdot \mathbf{k}}{\left(h_{T}^{2}+2\left(k^{-}\right)^{2}\right)\left(k_{T}^{2}+2\left(k^{-}\right)^{2}\right)} \\
& \left.+\frac{k_{T}^{2}}{\left(k_{T}^{2}+2\left(k^{-}\right)^{2}\right)^{2}}\right]
\end{aligned}
$$

where $2\left(k^{-}\right)^{2}=k_{T}^{2} e^{-2 y}$. Very schematically, one could describe the terms in Eq. (62) as follows. The first term is the square of the amplitude of a process in which the target quark emits a gluon of momentum $\mathbf{h}$ which then goes through the nuclear sheet, picks up a momentum $\mathbf{k}-\mathbf{h}$ and produces a gluon of momentum $\mathbf{k}$. All momenta $\mathbf{h}$ are integrated over. This amplitude is the first term in Eq. (42) and it is what one would automatically write down, but this would lead to physically wrong conclusions. For example, when one computes the leading term at large $k_{T}$, it could be negative for some rapidities. There is another process, in which the quark emits before the collision. This is the second term in Eq. (42) but with $u^{i}(\mathbf{x})$ replaced by $v^{i}(\mathbf{x})$, the initial state emission function ("emitting a gluon of momentum $\mathbf{k}$ " means taking the Fourier transform over $d^{2} x$ of this function). This process interferes with the first one and produces the second term in Eq. (62); its square gives the last term. Including all of these eliminates all nonphysicalities.

Let us then study the large $k_{T}$ behavior of Eq. (62) in the case of finite gluon rapidity $y$, which is $\mathcal{O}(1)$ in the target fragmentation region we are interested in. Applying again the expansion in Eq. (56) leads to

$$
\begin{aligned}
\left.\left(\frac{16 \pi^{3}}{g^{2} C_{F}}\right) \frac{d N}{d y d^{2} k}\right|_{\text {bulk }}= & +\frac{\left(Q_{s}^{A}\right)^{2}}{\pi m_{\mathrm{IR}}^{2} k_{T}^{2}\left(1+e^{-2 y}\right)^{2}}+\int \frac{d^{2} h}{(2 \pi)^{2}} \frac{4\left(Q_{s}^{A}\right)^{2}}{\left(|\mathbf{k}-\mathbf{h}|^{2}+m_{\mathrm{IR}}^{2}\right)^{2}} \\
& \times\left[\frac{h_{T}^{2}}{\left(h_{T}^{2}+k_{T}^{2} e^{-2 y}+m_{\mathrm{IR}}^{2}\right)^{2}}-\frac{2 \mathbf{h} \cdot \mathbf{k}}{\left(h_{T}^{2}+k_{T}^{2} e^{-2 y}+m_{\mathrm{IR}}^{2}\right)\left(k_{T}^{2}+k_{T}^{2} e^{-2 y}\right)}\right]+\mathcal{O}\left(\frac{\left(Q_{s}^{A}\right)^{2}}{k_{T}^{2}}\right),
\end{aligned}
$$

where the first term in the rhs of Eq. (63) arises from the trivial $h$-integration over the constant $k_{T}^{2} /\left(k_{T}^{2}+k_{T}^{2} e^{-2 y}\right)^{2}$ contribution and the mass term $m_{\mathrm{IR}}$ is introduced as an infrared regulator. Of course, for small $y$, as long as $k_{T}^{2} e^{-2 y}>m_{\mathrm{IR}}^{2}$, it is not needed in the two last terms, but becomes important for

$$
y \geq \log \frac{k_{T}}{m_{\mathrm{IR}}},
$$

and, in fact, has important dynamical consequences for the $y$ distribution.

In order to get the full gluon spectrum at large $k_{T}$ we first carry out exactly the integrals over azimuthal angle and then over $h_{T}$ and finally expand in small $m_{\mathrm{IR}}$ [in the $\left(|\mathbf{k}-\mathbf{h}|^{2}+m_{\mathrm{IR}}^{2}\right)$ term]. This gives the result

$$
\left.\left(\frac{16 \pi^{3}}{g^{2} C_{F}}\right) \frac{d N}{d y d^{2} k}\right|_{\text {bulk }}=\frac{2}{\pi\left(1+e^{-2 y}\right)^{2}} \frac{\left(Q_{s}^{A}\right)^{2}}{k_{T}^{4}}\left[\frac{1}{2}\left(1+\tanh ^{2}(y)\right) \log \left(\frac{k_{T}^{2}\left(1+e^{-2 y}\right)}{m_{\mathrm{IR}} \sqrt{m_{\mathrm{IR}}^{2}+k_{T}^{2} e^{-2 y}}}\right)-\tanh ^{2}(y)\right]+\mathcal{O}\left(m_{\mathrm{IR}}^{2}, \frac{\left(Q_{s}^{A}\right)^{2}}{k_{T}^{2}}\right),
$$

where the powerlike divergence $\sim 1 /\left(k_{T} m_{\mathrm{IR}}\right)^{2}$ in Eq. (63) cancels out after the integration. As expected, we recover the standard perturbative $\sim 1 / k_{T}^{4}$ behavior with a logarithmic correction. The overall rapidity structure can be written in the more explicit form

$$
\begin{aligned}
\left.\left(\frac{16 \pi^{3}}{g^{2} C_{F}}\right) \frac{d N}{d y d^{2} k}\right|_{\text {bulk }}= & \frac{2\left(Q_{s}^{A}\right)^{2}}{\pi k_{T}^{4}} \frac{1}{\left(1+e^{-2 y}\right)^{2}} \\
& \times\left[\frac{1}{2}\left(1+\tanh ^{2}(y)\right)\left(y+\log \left(1+e^{-2 y}\right)-\frac{1}{2} \log \left(1+\frac{m_{\mathrm{IR}}^{2}}{k_{T}^{2}} e^{2 y}\right)+\log \frac{k_{T}}{m_{\mathrm{IR}}}\right)-\tanh ^{2}(y)\right] .
\end{aligned}
$$




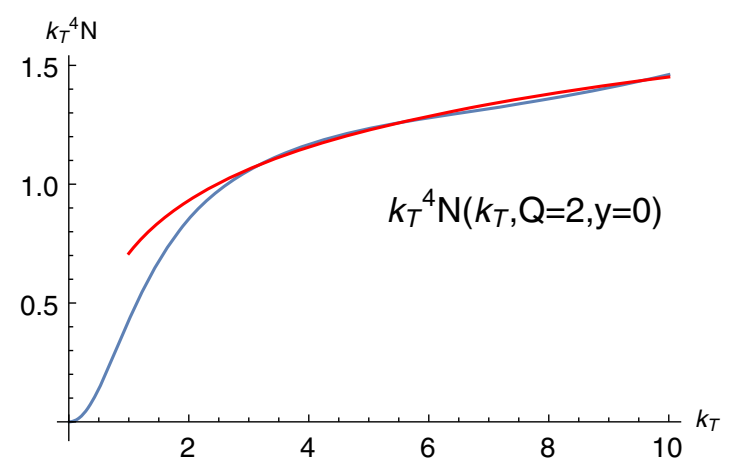

FIG. 2. A plot of $k_{T}^{4} N\left(k_{T}, y=0\right)$ computed from Eq. (67) for $y=0$ and for the correlator $S$ computed for $Q_{s}^{A}=2$ and $m_{\mathrm{IR}}=0.2$. The red curve is the logarithmic approximation in Eq. (69).

This expression emphasizes the fact that at small $y<$ $\log \left(k_{T} / m_{\mathrm{IR}}\right)$ the distribution has a dominant linear increase in $y$, which above this turns to a plateau.

Consider then full numerical evaluation of the bulk result in Eq. (62). To evaluate the $h$-integral we first make the change of variables, $\mathbf{h}=\mathbf{h}-\mathbf{k}$, and do analytically the azimuthal integral around $\mathbf{k}$. The $\mathbf{h}$ integral in Eq. (62) becomes

$$
\left.\left(\frac{16 \pi^{3}}{g^{2} C_{F}}\right) \frac{d N}{d y d^{2} k}\right|_{\text {bulk }}=2 \int_{0}^{\infty} d \hat{h}_{T} \frac{\hat{h}_{T} S\left(k_{T} \hat{h}_{T}\right)}{2 \pi} w_{\text {bulk }}\left[\hat{h}_{T}, y\right],
$$

where the variable $k_{T}$ has been scaled out by using $h_{T} \equiv k_{T} \hat{h}_{T}$ and the weight function $w$ is given by

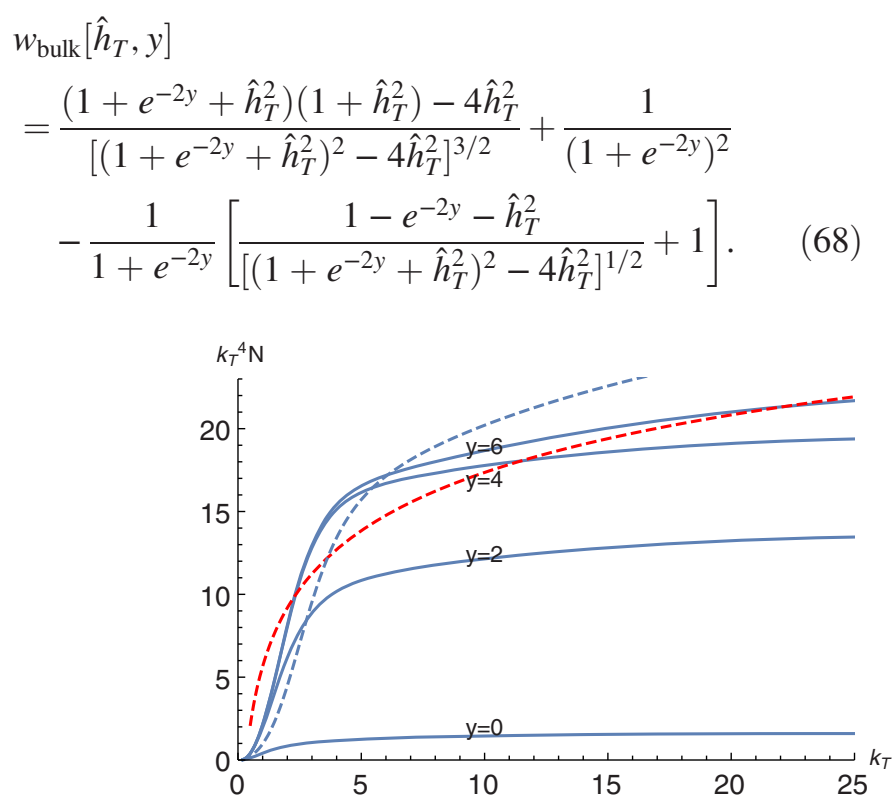

The expression in Eq. (67) can be integrated numerically by using Eq. (B18) to numerically compute the Fourier transform $S\left(q_{T}\right)$ of the correlation function $S\left(r_{T}\right)$. For $y=0$ this yields the $k_{T}$ spectrum shown in Fig. 2. Curves always refer to the integral in the right-hand side of Eq. (67), without the factors $g^{2} C_{F}$. From Fig. 2, one observes scaling with $k_{T}^{4}$, but also a suggestion of logarithmic variation. This can be fitted by

$$
0.322 \log \left(k_{T} / 2\right)+0.932
$$

which is plotted in red in Fig. 2. This quantitatively agrees with the logarithmic factor

$$
\frac{1}{\pi} \log \left(2 k_{T} / m_{\mathrm{IR}}\right) \sim 0.318 \log \left(k_{T} / 2\right)+0.953
$$

from Eq. (65) with $Q_{s}^{A}=2, y=0$ and $m_{\mathrm{IR}}=0.2$.

In Fig. 3 we show (left panel) the $k_{T}^{4}$-scaled $k_{T}$ spectrum of gluons as a function of $k_{T}$ for various fixed rapidities $y=0,1,2,4,6$ or (right panel) as a function of $y$ plotted for $k_{T}=2,10$, together with the large $k_{T}$ approximation (66) (red dashed curves in both panels).

The rapidity distribution in the fragmentation region shows first at small $y$ some structure which is well reproduced by the approximation Eq. (66), then a quasilinear increase which at larger $y$ turns to a plateau. The key factor producing this pattern is the scale of the IR log in Eq. (65). At small $y$ the IR divergence of the $1 /\left(h_{T}^{2}+\right.$ $\left.2\left(k^{-}\right)^{2}\right)$ term is cut off by $2\left(k^{-}\right)^{2}=k_{T}^{2} e^{-2 y}$. This disappears at large $y$ and the boundary clearly is when it decreases below $m_{\mathrm{IR}}^{2}$; i.e., IR screening by $m_{\mathrm{IR}}^{2}$ in all terms is effective [compare Eq. (65)] as long as

$$
y \leq \log \frac{k_{T}}{m_{\mathrm{IR}}} .
$$

We later identify this as the lower limit of the bremsstrahlung contribution. We do not see evidence of an upper limit of the fragmentation region; there is just the merging into

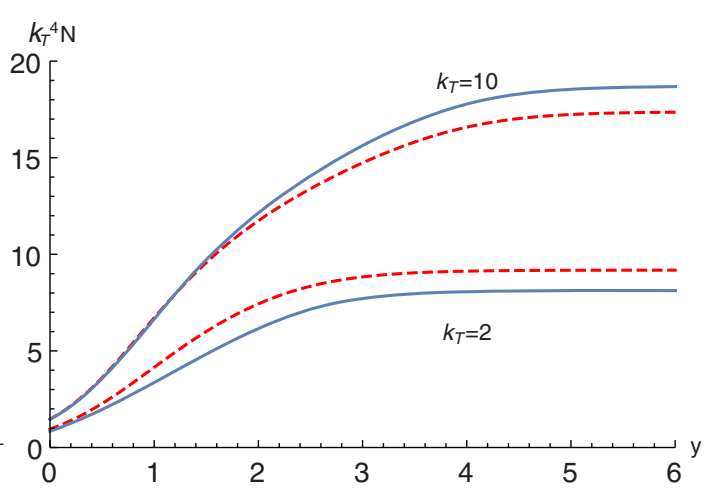

FIG. 3. A plot of $k_{T}^{4} N\left(k_{T}, y\right)$ computed from Eq. (67) for values of $y$ marked in the figure and for $Q_{s}^{A}=2$ and $m_{\mathrm{IR}}=0.2$ (left panel). The red dashed curve is the analytic large $k_{T}$ approximation Eq. (66) for $y=6$. The blue dashed curve is the $y \rightarrow \infty$ limit Eq. (72). The right panel shows the $y$ dependence for $k_{T}=2,10$ and for the same $Q_{s}^{A}, m_{\mathrm{IR}}$. The red dashed curve is the analytic approximation in Eq. (66). The curves are proportional to $\left(Q_{s}^{A}\right)^{2}$. 

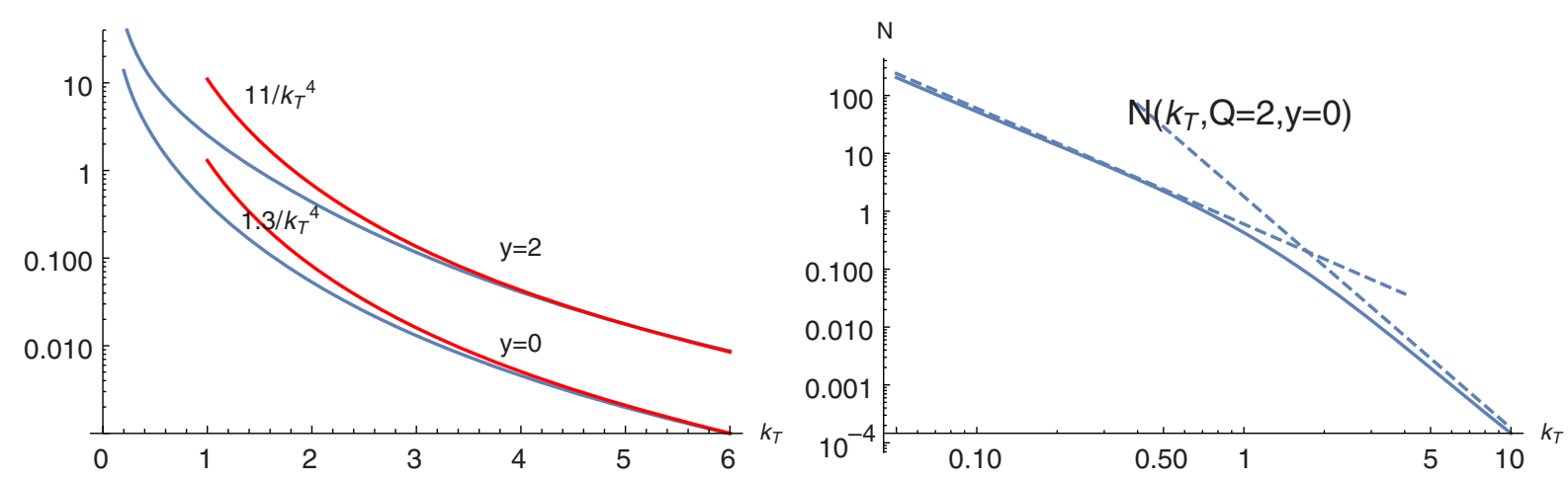

FIG. 4. A plot of unscaled $N\left(k_{T}, y\right)$ for small $k_{T}$ and $y=0,2$. The red curves are powerlike approximations const $/ k_{T}^{4}$. The right panel shows the data for $y=0$ on a $\log$-log scale so that a transition from $1.3 / k_{T}^{4}$ to $0.6 / k_{T}^{2}$ (dashed curves) is visible.

the central plateau. In contrast, we find that the bremsstrahlung contribution decays as $\sim e^{-2 y}$ when one approaches the central region [see Eq. (76)].

The limiting curve at large $y$ is simply obtained by taking this limit in the integrand or of the weight function Eq. (68). The result is

$\frac{2}{k_{T}^{2}} \int_{0}^{\infty} d h_{T} \frac{h_{T} S\left(h_{T}\right)}{2 \pi} \frac{h_{T}^{2}}{\left|k_{T}^{2}-h_{T}^{2}\right|}=\frac{2}{k_{T}^{2}} \int \frac{d^{2} h S\left(h_{T}\right)}{(2 \pi)^{2}} \frac{h_{T}^{2}}{\left|k_{T}^{2}-h_{T}^{2}\right|}$.

Regularizing the pole at $h_{T}=k_{T}$ in the range of integration by replacing the denominator above by $\sqrt{\left(k_{T}-h_{T}\right)^{2}+\left(Q_{s}^{A}\right)^{4}}, Q_{s}^{A}=2$, one obtains the central region $k_{T}$ distribution, multiplied by $k_{T}^{4}$, plotted in Fig. 3. One sees that in the target fragmentation region there is a slight enhancement relative to the $k_{T}$ distribution in the central region. The details of the computation, especially the extrapolation of the central region distribution this far in $y$, are very uncertain. Phenomenologically, by multiplying by $k_{T}$, this would give an estimate of how much a single quark contributes to energy deposition in the target fragmentation region.

In Fig. 4, we show the numerically computed gluon distribution without $k_{T}$ scaling so that one sees what happens in the saturation region, $k_{T} \ll Q_{s}^{A}=2$. The perturbative $1 / k_{T}^{4}$ behavior at large $k_{T}$ slows to a $1 / k_{T}^{2}$ behavior, but this persists down to $k_{T} \rightarrow 0$ since there is no target saturation scale. In fact, this is due to the second term in Eq. (62), which is precisely the ED-like radiation from quark acceleration. In a study of $p+A$ collisions there, qualitatively, would be a second saturation scale $Q^{p}<Q_{s}^{A}$ which would shield the $1 / k_{T}^{2}$ behavior at small $k_{T}$.

\section{B. Bremsstrahlung and its interference with bulk}

We have above split the general result Eq. (59) in the bulk part Eq. (60), which contains all nuclear effects and is independent of the assumed constant kick of the quark and the bremsstrahlung part Eq. (61), which does not contain nuclear effects, depends sensitively on the magnitude of the kick and is bounded from below (as we show) and above in rapidity. In fact, it is the same as classical ED radiation due to sudden acceleration of charged particle.

Inserting in Eq. (61) the variable $v_{T}=p_{T} / \sqrt{p_{T}^{2}+m^{2}}$ with

$$
\begin{aligned}
k_{T}^{2}+2\left(k^{-}\right)^{2} & =k_{T}^{2}\left(1+e^{-2 y}\right), \\
|\mathbf{k}-\xi \mathbf{p}|^{2} & =k_{T}^{2}\left(1-2 v_{T} e^{-y+y_{p}} \cos \phi+v_{T}^{2} e^{-2 y+2 y_{p}}\right),
\end{aligned}
$$

and

$$
\begin{aligned}
|\mathbf{k}-\xi \mathbf{p}|^{2}+\xi^{2} m^{2} & =k_{T}^{2}\left(1-2 v_{T} e^{-y+y_{p}} \cos \phi+e^{-2 y+2 y_{p}}\right), \\
\mathbf{k} \cdot(\mathbf{k}-\xi \mathbf{p}) & =k_{T}^{2}\left(1-v_{T} e^{-y+y_{p}} \cos \phi\right),
\end{aligned}
$$

gives the result

$$
\begin{aligned}
& \left.\left(\frac{16 \pi^{3}}{g^{2} C_{F}}\right) \frac{d N}{d y d^{2} k}\right|_{\text {bremss }} \\
& =\frac{2}{k_{T}^{2}}\left[\frac{1}{\left(1+e^{-2 y}\right)^{2}}+\frac{1-2 v_{T} e^{-\left(y-y_{p}\right)} \cos \phi+v_{T}^{2} e^{-2\left(y-y_{p}\right)}}{\left(1-2 v_{T} e^{-\left(y-y_{p}\right)} \cos \phi+e^{-2\left(y-y_{p}\right)}\right)^{2}}\right. \\
& \left.\quad-\frac{2\left(1-v_{T} e^{-\left(y-y_{p}\right)} \cos \phi\right)}{\left(1+e^{-2 y}\right)\left(1-2 v_{T} e^{-\left(y-y_{p}\right)} \cos \phi+e^{-2\left(y-y_{p}\right)}\right)}\right]
\end{aligned}
$$

where we have defined $\cos \phi \equiv \mathbf{k} \cdot \mathbf{p} /\left(k_{T} p_{T}\right)$. From Eq. (75) one sees that a factor $1 / k_{T}^{2}$ scales out of $\left|\mathcal{M}_{\text {brems }}\right|^{2}$, and with fixed kick the entire bremsstrahlung gluon distribution is $1 / k_{T}^{2}$ times a function of $y, y_{p}, p_{T}, \phi$ and $m$. For small $m$ the entire contribution of Eq. (75) to the gluon spectrum simplifies to (note the extra factor 2)

$$
\begin{gathered}
\left.\left(\frac{16 \pi^{3}}{g^{2} C_{F}}\right) \frac{d N}{d y d^{2} k}\right|_{\text {bremss, } m=0} \\
=\frac{1}{2 k_{T}^{2}} \frac{\cosh \left(y+y_{p}\right)+\cos \phi}{\cosh ^{2} y\left(\cosh \left(y-y_{p}\right)-\cos \phi\right)} .
\end{gathered}
$$

At large $y$ this decreases $\sim e^{-2 y}$, which implies that bremsstrahlung contribution vanishes in the central region. 
In the denominator one has $p_{\text {in }} \cdot k=-m k_{T} \cosh y, p \cdot k=$ $-k_{T} p_{T}\left(\cosh \left(y-y_{p}\right)-\cos \phi\right)$ so that there is a collinear singularity for emission parallel to the kicked quark. This is shielded by finite quark mass $m$. We are mainly interested in $y$ dependence for $y$ close to 0 and for $y_{p}$ large, large recoil. Its magnitude then is order $1 / k_{T}^{2}$; there is no dependence on the nucleus so no nuclear enhancement by a factor $\left(Q_{s}^{A}\right)^{2}$ like for the bulk contribution. Even more importantly, the general dependence $\sim 1 / k_{T}^{2}$ cannot hold to very large $k_{T}$ : ultimately a gluon propagator squared $\sim 1 / k_{T}^{4}$ must enter. The source of this deficiency at large $k_{T}$ is the assumption of constant kick or constant momentum transfer from the nucleus.

There are kinematic constraints on the process

$$
\begin{aligned}
P_{A}+p_{\text {in }} & =P_{X}+p+k, \quad P_{A}=P_{X}+q, \\
q+p_{\text {in }} & =p+k
\end{aligned}
$$

studied. Since in the thin sheet limit $P_{A}=\left(P_{A}^{+} \rightarrow \infty, 0, \mathbf{0}\right)$ we have also $P_{X}^{+} \rightarrow \infty$ from conservation of the + component. Then the minus components $P_{A}^{-}=M_{A}^{2} /\left(2 P_{A}^{+}\right)$and $P_{X}^{-}=$ $M_{X}^{2} /\left(2 P_{X}^{+}\right) \approx-q^{-}$are also very small. For usual mass shell particles $q^{-}$is $\leq 0$. The conservation rules of minus and transverse components become

$$
q^{-}+\frac{m}{\sqrt{2}}=p^{-}+k^{-}, \quad \mathbf{q}=\mathbf{p}+\mathbf{k}=-\mathbf{P}_{\mathrm{X}} .
$$

For the minus components

$$
\sqrt{2} q^{-}+m=k_{T} e^{-y}+m_{T} e^{-y p} .
$$

The rhs is positive so sufficiently large negative $q^{-}$can even make the process impossible for on-shell particles. Assuming $q^{-}=0$ one can solve

$$
y=\log \frac{k_{T}}{m-m_{T} e^{-y_{p}}}>\log \frac{k_{T}}{m} .
$$

We are thus back to Eq. (71) and see that the lower limit of bremsstrahlung is approximately the same as the estimated lower limit of the rapidity plateau of the bulk distribution in the MV model. To reach large $k_{T}$ one must go to the central region, $y \gg 1$. Conversely, at fixed rapidity, there is an upper limit to $k_{T}, k_{T}<m e^{y}$. This cuts off the $1 / k_{T}^{2}$ distribution.

The bulk contribution, i.e., the MV model, produces in the fragmentation region the increasing distribution in Fig. 3, proportional to $\left(Q_{s}^{A}\right)^{2}$ at large $k_{T}$. Due to many uncertainties, the detailed comparison of relative magnitudes at large $k_{T}$ will have to wait for a definite model of improved bremsstrahlung. Anyway bremsstrahlung decays $\sim e^{-2 y}$ with increasing $y$ and in the central region the gluonic bulk dominates.

\section{Interference contribution}

Let us then consider the interference term between the bulk and bremsstrahlung contributions

$$
\begin{aligned}
& \left.\left(\frac{16 \pi^{3}}{g^{2} C_{F}}\right) \frac{d N}{d y d^{2} k}\right|_{\text {interf }} \\
& \equiv 4 \int \frac{d^{2} h}{(2 \pi)^{2}} S(\mathbf{k}-\mathbf{h})\left[\frac{h^{i}}{h_{T}^{2}+2\left(k^{-}\right)^{2}}-\frac{k^{i}}{k_{T}^{2}+2\left(k^{-}\right)^{2}}\right] \\
& \quad \times\left[\frac{k^{i}}{k_{T}^{2}+2\left(k^{-}\right)^{2}}-\frac{k^{i}-\xi p^{i}}{|\mathbf{k}-\xi \mathbf{p}|^{2}+\xi^{2} m^{2}}\right] .
\end{aligned}
$$

To do the transverse momentum $h_{T}$ integral numerically and to do one angular integral analytically, we have to switch the convolution factor $\mathbf{k}-\mathbf{h}$ to the brackets so that the integral to be done becomes

$$
\begin{aligned}
\left.\left(\frac{16 \pi^{3}}{g^{2} C_{F}}\right) \frac{d N}{d y d^{2} k}\right|_{\text {interf }}= & 4 \int \frac{d^{2} h}{(2 \pi)^{2}} S(\mathbf{h})\left[\frac{k_{T}^{2}-\mathbf{h} \cdot \mathbf{k}}{\left(|\mathbf{k}-\mathbf{h}|^{2}+a^{2}\right)\left(k_{T}^{2}+a^{2}\right)}\right. \\
& \left.-\frac{k_{T}^{2}-\mathbf{h} \cdot \mathbf{k}-\xi(\mathbf{k} \cdot \mathbf{p}-\mathbf{h} \cdot \mathbf{p})}{\left(|\mathbf{k}-\mathbf{h}|^{2}+a^{2}\right)\left(\kappa^{2}+\xi^{2} m^{2}\right)}-\frac{k_{T}^{2}}{\left(k_{T}^{2}+a^{2}\right)^{2}}+\frac{k_{T}^{2}-\xi \mathbf{k} \cdot \mathbf{p}}{\left(k_{T}^{2}+a^{2}\right)\left(\kappa^{2}+\xi^{2} m^{2}\right)}\right],
\end{aligned}
$$

where we abbreviated $\kappa^{2} \equiv|\mathbf{k}-\xi \mathbf{p}|^{2}, a^{2} \equiv 2\left(k^{-}\right)^{2}$ and the variable $\xi=\left(k_{T} / m_{T}\right) e^{-y+y_{p}}$. The integration over the angle between $\mathbf{k}$ and $\mathbf{h}$ can now be carried out and the integral yields

$$
\begin{aligned}
\left.\left(\frac{16 \pi^{3}}{g^{2} C_{F}}\right) \frac{d N}{d y d^{2} k}\right|_{\text {interf }}= & 2 \int_{0}^{\infty} d h_{T} \frac{h_{T} S\left(h_{T}\right)}{2 \pi}\left[\frac { k _ { T } ^ { 2 } - a ^ { 2 } - h _ { T } ^ { 2 } + \sqrt { K } } { \sqrt { K } } \left\{\frac{1}{k_{T}^{2}+a^{2}}\right.\right. \\
& \left.\left.-\frac{1}{\kappa^{2}+\xi^{2} m^{2}}\left(1-\frac{p_{T}}{k_{T}} \xi \cos (\phi)\right)\right\}-\frac{2 k_{T}^{2}}{\left(k_{T}^{2}+a^{2}\right)^{2}}+\frac{2 k_{T}^{2}-2 \xi k_{T} p_{T} \cos (\phi)}{\left(k_{T}^{2}+a^{2}\right)\left(\kappa^{2}+\xi^{2} m^{2}\right)}\right],
\end{aligned}
$$

where we have defined the factor $K$ as

$$
K \equiv\left(a^{2}+k_{T}^{2}+h_{T}^{2}\right)^{2}-4 k_{T}^{2} h_{T}^{2}
$$


Using the kinematics introduced in Eqs. (73) and (74) and scaling out the variable $k_{T}$ by denoting $h_{T}=k_{T} \hat{h}_{T}$ the expression in Eq. (83) simplifies to

$$
\left.\left(\frac{16 \pi^{3}}{g^{2} C_{F}}\right) \frac{d N}{d y d^{2} k}\right|_{\text {interf }}=2 \int_{0}^{\infty} d \hat{h}_{T} \frac{\hat{h}_{T} S\left(k_{T} \hat{h}_{T}\right)}{2 \pi} \omega_{\text {interf }}\left[\hat{h}_{T} ; v_{T}, y, y_{p}, \phi\right],
$$

where the weight function $\omega_{\text {interf }}$ is given by

$$
\begin{aligned}
\omega_{\text {interf }}\left[\hat{h}_{T} ; v_{T}, y, y_{p}, \phi\right]= & {\left[\frac{1-e^{-2 y}-\hat{h}_{T}^{2}}{\left[\left(1+e^{-2 y}+\hat{h}_{T}^{2}\right)^{2}-4 \hat{h}_{T}^{2}\right]^{1 / 2}}-\tanh (y)\right] } \\
& \times\left\{\frac{1}{1+e^{-2 y}}-\frac{1-v_{T} e^{-\left(y-y_{p}\right)} \cos \phi}{1-2 v_{T} e^{-\left(y-y_{p}\right)} \cos \phi+e^{-2\left(y-y_{p}\right)}}\right\} .
\end{aligned}
$$

\section{Sum of bulk, bremsstrahlung and interference contributions}

Finally, we calculate numerically the total gluon distribution for the process $q+A \rightarrow X+q+g$. This is obtained either directly computing the main result in Eq. (53) or by summing the bulk, bremsstrahlung and interference contributions in Eqs. (67), (75) and (85):

$$
\left.\left(\frac{16 \pi^{3}}{g^{2} C_{F}}\right) \frac{d N}{d y d^{2} k}\right|_{\text {total }} \equiv\left(\frac{16 \pi^{3}}{g^{2} C_{F}}\right)\left[\left.\frac{d N}{d y d^{2} k}\right|_{\text {bulk }}+\left.\frac{d N}{d y d^{2} k}\right|_{\text {bremss }}+\left.\frac{d N}{d y d^{2} k}\right|_{\text {interf }}\right]
$$

The total result in Eq. (87) can be rewritten in a very compact form

$$
\begin{aligned}
\left.\left(\frac{16 \pi^{3}}{g^{2} C_{F}}\right) \frac{d N}{d y d^{2} k}\right|_{\text {total }}= & 2 \int_{0}^{\infty} d h_{T} \frac{h_{T} S\left(h_{T}\right)}{2 \pi} \frac{1}{k_{T}^{2}}\left[\frac{k_{T}^{2}\left[\left(h_{T}^{2}+k_{T}^{2}+a^{2}\right)\left(h_{T}^{2}+k_{T}^{2}\right)-4 k_{T}^{2} h_{T}^{2}\right]}{K^{3 / 2}}\right. \\
& \left.+\frac{1-v_{T} e^{\Delta y} \cos \phi}{1-2 v_{T} e^{\Delta y} \cos \phi+e^{2 \Delta y}}\left(\frac{h_{T}^{2}-k_{T}^{2}+a^{2}}{K^{1 / 2}}-1\right)+\frac{1-2 v_{T} e^{\Delta y} \cos \phi+v_{T}^{2} e^{2 \Delta y}}{\left(1-2 v_{T} e^{\Delta y} \cos \phi+e^{2 \Delta y}\right)^{2}}\right],
\end{aligned}
$$

where $\Delta y=y_{p}-y, a^{2}=k_{T}^{2} e^{-2 y}$ and the factor $K$ is defined in Eq. (84).

In Fig. 5 (left panel) we show the numerically computed and $k_{T}$-scaled (though for $k_{T}=1$ this has no effect) total gluon distribution as a function of $y$ for fixed values of $k_{T}$, $p_{T}, y_{p}$ and $\phi$. The right panel shows the same distribution as a function of $y_{p}$ for fixed values of $k_{T}, p_{T}, y$ and $\phi$. The quark mass is always fixed to $m=0.2$ and $Q_{s}^{A}=2$. In both
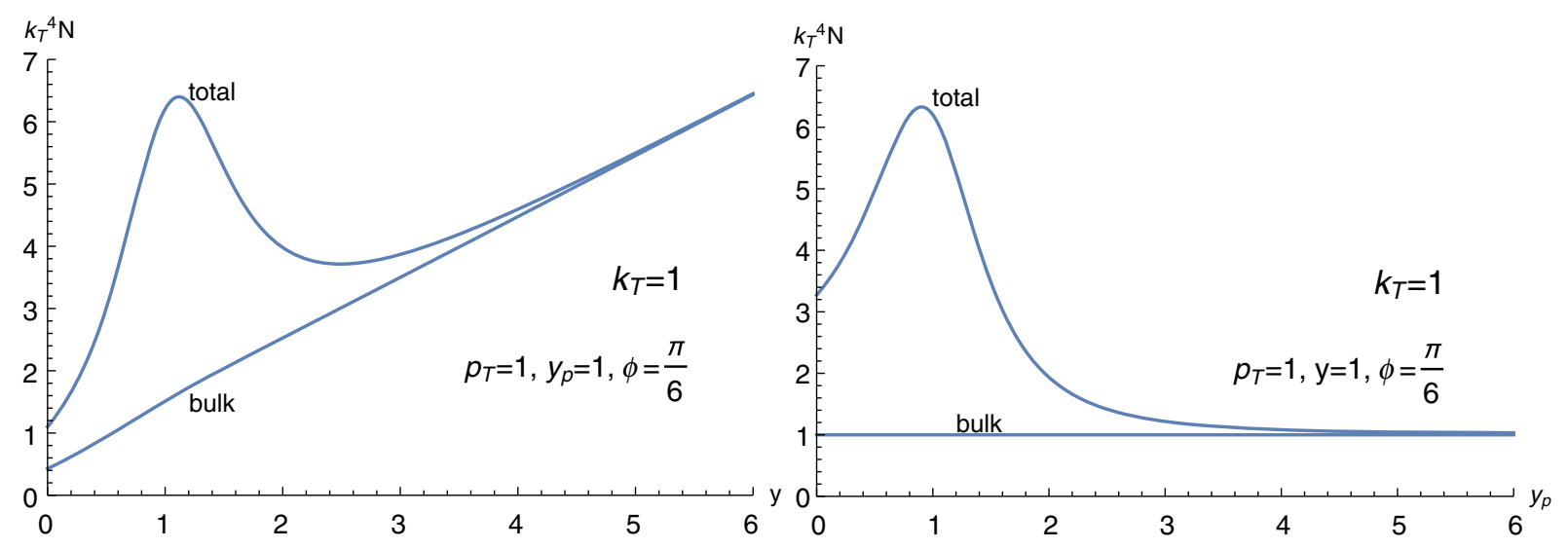

FIG. 5. A plot (left panel) of scaled total gluon distribution Eq. (53) as a function of $y$ for fixed $k_{T}, p_{T}, y_{p}$ and $\phi$. The curve marked bulk is the $k_{T}=1$ rapidity distribution computed from (62) (cf. Fig. 3). The right panel is the same distribution but now plotted as a function of $y_{p}$ for fixed $k_{T}, p_{T}, y$ and $\phi$. Here the only reference is that we know that bulk is independent of recoil parameters. 
panels the baseline is given by the bulk curve which should be compared with those in Fig. 3: at this small $k_{T}$ one does not observe any turnover to the central plateau. On top of the bulk curve one observes a strong peak structure from bremsstrahlung and its interference with bulk. The detailed form of the peak is sensitively dependent on the relative magnitudes of $\mathbf{k}$ and the recoil momentum $\mathbf{p}$. If $\phi=0$, one can even hit the $p \cdot k=0$ singularity at $y=y_{p}$. In the figure $\phi=\pi / 6$ and increasing it further the peak structure gets less dominant. This peak structure is a definite experimental prediction of the model, which, of course, is modified by quantum mechanics and the fact that the quark in practice has to be embedded in a hadron.

\section{CONCLUSIONS}

We have in this paper studied QCD dynamics in the usual CGC formalism but in the rather unusual setting of a very large energy nucleus colliding with a static classical colored particle, called a quark. The collision kicks the initial quark to some constant final momentum $p^{\mu}$. Physically one has in mind a study of nucleus-nucleus collisions in the rest frame of either of the nuclei, i.e., in the target or beam fragmentation regions. $*$ The rationale is that it might be useful to dissect the problem in microscopic pieces and the extreme clearly is to just have a single quark as a target. From this one can then proceed to the level of nucleons and finally nuclei.

The outcome is a rather compact formula describing the final gluonic radiation field, from which the distribution of gluons is computed by complex squaring and averaging over an ensemble of sources. The result can be split in two components. The first, the bulk, arises mainly from the interaction of the nuclear color field with the color electric field of the nucleus, is independent of the momentum of the kicked quark and gives the gluon $k_{T}$ and longitudinal rapidity $y$ distribution in the range we are interested in. At fixed $k_{T}$ the $y$ distribution grows monotonically from negative values of $y$ up to $y \approx \log \left(k_{T} / m_{\text {quark }}\right)$ where it levels off to a central region plateau. The second component, bremsstrahlung, is in the classical approximation independent of interactions with the nucleus, depends on the kicked quark momentum $p^{\mu}$ and decreases too slowly with increasing $k_{T}$. At very large $k_{T}$ it has to be improved by including recoil effect in the scattered quark. However, at smaller $k_{T} \ll Q_{s}^{A}$, where most of the gluon production takes place, it should give a good description of physics. We find that at rapidities in the target framentation region it gives rise to a significant peak, somewhat dependent on the recoil momentum. At large $y$ bremsstrahlung decays $\sim e^{-2 y}$ and thus the bulk contribution dominates in the central region.

The outcome should now be usable to estimate energy deposition from gluon radiation in the target fragmentation region, as input to initial conditions of hydrodynamical evolution of QCD matter formed. Baryon number initial values could come from the Dirac equation solutions in [24]. However, the inclusion of recoil effects in the classical approximation is still incomplete. One obvious avenue for progress is computing joint production rates of gluons and quarks by extending the computation in [15] to the target fragmentation region.

\section{ACKNOWLEDGMENTS}

R. P. is supported by the European Research Council, Grant No. 725369, and L. M. was supported by the U.S. DOE under Grant No. DE-FG02-00ER41132. K. K. and R. P. thank F. Gelis, T. Lappi, N. Armesto and W. Van Der Schee for discussions.

\section{APPENDIX A: GREEN'S FUNCTION INTEGRALS OVER THE CLASSICAL QUARK CURRENTS}

In this section, we derive the results used in Eq. (36). Starting from the most difficult case, the task is to compute the advanced Green's function integral towards the line $x^{-}=0$ over the scattered quark (see Fig. 6),

$a_{\text {fin }}^{i}\left(x^{+}, x^{-}=0, \mathbf{x}\right) \equiv \int d^{4} y G_{A}(x-y) \mathcal{J}_{\text {eff }}^{i}\left(y^{+}, y^{-}>0, \mathbf{y}\right)$.

From the derivative of this with respect to $x^{+}$its Fourier transform over the $x^{+}$can be computed,

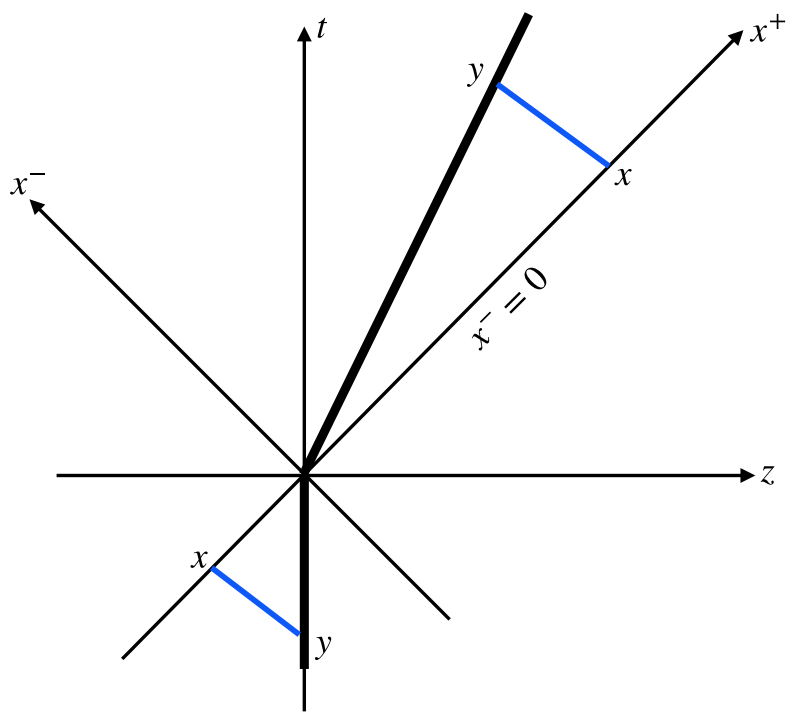

FIG. 6. Computation of the two pieces (A1) and (A14) of the radiation field along $x^{-}=0$. The first piece is sourced by the kicked quark and brought to $x^{-}=0$ by the advanced Green's function and the second is sourced by the initial quark and brought to $x^{-}=0$ by the retarded Green's function. 


$$
u^{i}(\mathbf{x}) \equiv 2 i \int d x^{+} e^{i k^{-} x^{+}} \partial_{+} a_{\mathrm{fin}}^{i}\left(x^{+}, x^{-}=0, \mathbf{x}\right)
$$

where the advanced Green's function in the LC coordinates is given by

$$
G_{A}(x-y)=+\frac{1}{2 \pi} \theta\left(x^{-}-y^{-}\right) \delta\left((x-y)^{2}\right)
$$

and the source term $\mathcal{J}_{\text {eff }}^{i}\left(y^{-}>0\right)$ [defined in Eq. (29)] corresponds to the kicked quark current Eq. (16),

$$
\begin{aligned}
\mathcal{J}_{\text {eff }}^{i}\left(y^{+}, y^{-}>0, \mathbf{y}\right)= & \left(\frac{p^{i}}{p^{-}}+\partial^{i} \frac{1}{\partial_{+}}\right) \delta\left(y^{+}-\frac{p^{+}}{p^{-}} y^{-}\right) \\
& \times \delta^{(2)}\left(\mathbf{y}-\frac{\mathbf{p}}{p^{-}} y^{-}\right)
\end{aligned}
$$

Since the source term in Eq. (A4) contains the inverse of the operator $\partial_{+}$, it is actually simpler to solve for $\partial_{+} a_{\text {fin. }}^{i}$. Also the Fourier transform of $\partial_{+} a_{\text {fin }}^{i}$ in the $x^{+}$direction converges, not that of $a_{\text {fin }}^{i}$ as such. Inserting these explicitly into Eq. (A1) yields

$$
\begin{aligned}
2 \pi \partial_{+} a_{\text {fin }}^{i}\left(x^{+}, x^{-}, \mathbf{x}\right)= & \int d y^{+} d y^{-} d^{2} y \theta\left(y^{-}-x^{-}\right) \\
& \times \delta\left((x-y)^{2}\right)\left(\frac{p_{i}}{p^{-}} \partial_{+}^{y}+\partial_{i}^{y}\right) \\
& \times \delta\left(y^{+}-\frac{p^{+}}{p^{-}} y^{-}\right) \delta^{(2)}\left(\mathbf{y}-\frac{\mathbf{p}}{p^{-}} y^{-}\right) .
\end{aligned}
$$

The derivative of the delta functions can be dealt with by partial integration by moving the derivative bracket two steps to the left (with sign change) and then converting $\partial^{y}$ to $-\partial^{x}$ (since the integrand is a function of $y-x$ ) and taking it outside the integral. Using the other delta functions the light-cone delta function becomes a quadratic polynomial in $y^{+}$,

$$
\begin{aligned}
\delta\left((x-y)^{2}\right) & =\delta\left(\frac{p^{2}}{\left(p^{+}\right)^{2}}\left(y^{+}-y_{+}^{+}\right)\left(y^{+}-y_{-}^{+}\right)\right) \\
y_{ \pm}^{+} & =-\frac{p^{+}}{m^{2}}\left(p \cdot x \pm \sqrt{(p \cdot x)^{2}+m^{2} x^{2}}\right)
\end{aligned}
$$

The scalar products here are four dimensional. On the interesting line $x^{-}=0$ we have $x^{2}=x_{T}^{2}$; the two roots have opposite signs so that only one of them contributes (Fig. 6 shows that $y^{+}>0$ ). Collecting all factors, the result is

$$
\begin{aligned}
& \partial_{+} a_{\text {fin }}^{i}\left(x^{+}, 0, \mathbf{x}\right) \\
& =\frac{1}{4 \pi}\left(p_{i} \partial_{+}+p^{-} \partial_{i}\right) \frac{1}{\sqrt{\left(-p^{-} x^{+}+\mathbf{p} \cdot \mathbf{x}\right)^{2}+m^{2} x_{T}^{2}}} \\
& =-\frac{1}{4 \pi} m^{2} p^{-} \frac{x^{i}}{\left[\left(-p^{-} x^{+}+\mathbf{p} \cdot \mathbf{x}\right)^{2}+m^{2} x_{T}^{2}\right]^{3 / 2}} .
\end{aligned}
$$

Substituting this into Eq. (A2) we find

$$
\begin{aligned}
u^{i}(\mathbf{x})= & -\frac{i x^{i}}{2 \pi} m^{2} p^{-} \\
& \times \int d x^{+} \frac{e^{i k^{-} x^{+}}}{\left[\left(p^{-}\right)^{2}\left(x^{+}-\frac{1}{p^{-}} \mathbf{p} \cdot \mathbf{x}\right)^{2}+m^{2} x_{T}^{2}\right]^{3 / 2}},
\end{aligned}
$$

where the integrand is written in a form which suggests replacing $x^{+}$by

$$
z^{+}=x^{+}-\frac{1}{p^{-}} \mathbf{p} \cdot \mathbf{x}
$$

This also creates a transverse momentum shift $k_{i} \rightarrow k_{i}-$ $\frac{k^{-}}{p^{-}} p_{i}$ in the $\mathbf{x}$ integral. Integration over $z^{+}$is straightforward and gives the result

$u^{i}(\mathbf{x})=-\frac{i}{2 \pi} \frac{2 m k^{-}}{p^{-}} \frac{x^{i}}{x_{T}} \exp \left[i \frac{k^{-}}{p^{-}} \mathbf{p} \cdot \mathbf{k}\right] K_{1}\left(\frac{m k^{-}}{p^{-}} x_{T}\right)$.

Furthermore, we also need the Fourier transform of $u^{i}(\mathbf{x}) \rightarrow u^{i}(\mathbf{k})$. The integration over the two-dimensional transverse space can be easily performed by first noting that

$$
\int d^{2} x e^{-i \mathbf{k} \cdot \mathbf{x}} x^{i} f(\mathbf{x})=-2 i \pi \frac{k^{i}}{k_{T}} \int_{0}^{\infty} d x_{T} x_{T}^{2} f(\mathbf{x}) J_{1}\left(k_{T} x_{T}\right),
$$

where $J_{1}$ is the modified Bessel function of the second kind. This yields the expression

$$
\begin{aligned}
\int d^{2} x e^{-i \mathbf{k} \cdot \mathbf{x}} u^{i}(\mathbf{x})= & -\frac{2 m k^{-}}{p^{-}} \frac{r^{i}}{r_{T}} \int_{0}^{\infty} d x_{T} x_{T} K_{1}\left(\frac{m k^{-}}{p^{-}} x_{T}\right) \\
& \times J_{1}\left(r_{T} x_{T}\right),
\end{aligned}
$$

where we have defined $r^{i}=k^{i}-\left(k^{-} / p^{-}\right) p^{i}$. Using this result and performing the remaining integral we find

$$
u^{i}(\mathbf{k})=\frac{-2\left(k^{i}-\frac{k^{-}}{p^{-}} p^{i}\right)}{\left|k^{i}-\frac{k^{-}}{p^{-}} p^{i}\right|^{2}+\left(\frac{k^{-}}{p^{-}}\right)^{2} m^{2}} .
$$

Let us then consider the easier case, the retarded Green's function integral towards the line $x^{-}=0$ over the initial static quark (see Fig. 6) 
$a_{\mathrm{ini}}^{i}\left(x^{+}, x^{-}=0, \mathbf{x}\right) \equiv \int d^{4} y G_{R}(x-y) \mathcal{J}_{\mathrm{eff}}^{i}\left(y^{+}, y^{-}<0, \mathbf{y}\right)$.

From this one can then compute the other function in Eq. (36),

$$
v^{i}(\mathbf{x}) \equiv 2 i \int d x^{+} e^{i k^{-} x^{+}} \partial_{+} a_{\mathrm{ini}}^{i}\left(x^{+}, x^{-}=0, \mathbf{x}\right),
$$

where the retarded Green's function is given by

$$
G_{R}(x-y)=+\frac{1}{2 \pi} \theta\left(y^{-}-x^{-}\right) \delta\left((x-y)^{2}\right) .
$$

The source term $\mathcal{J}_{\text {eff }}^{i}\left(y^{-}<0\right)$ corresponding to the initial static quark reads

$$
\mathcal{J}_{\text {eff }}^{i}\left(y^{+}, y^{-}<0, \mathbf{y}\right)=\partial^{i} \frac{1}{\partial_{+}} \delta\left(y^{+}-y^{-}\right) \delta^{(2)}(\mathbf{y}) .
$$

Following the same steps as before we find

$$
v^{i}(\mathbf{x})=-\frac{i}{2 \pi} 2 \sqrt{2} k^{-} \frac{x^{i}}{x_{T}} K_{1}\left(\sqrt{2} k^{-} x_{T}\right) .
$$

Fourier transforming this to momentum space gives [this follows from Eq. (A13) with $p_{i}=0$ and $p^{-}=m / \sqrt{2}$ ]

$$
v^{i}(\mathbf{k})=\frac{-2 k^{i}}{k_{T}^{2}+2\left(k^{-}\right)^{2}} .
$$

\section{APPENDIX B: WILSON LINE CORRELATORS}

There is extensive literature on the calculation of the correlation function of Wilson lines; see for example $[10,31,32]$. In this section, we outline how to compute average over the color sources in the correlation function $S(\mathbf{x}-\mathbf{y})$ of two Wilson lines introduced in Eq. (44). All of this is well-known material, but the discussion in this paper relies so heavily on it that maybe it is useful to summarize its derivation.

To calculate gauge invariant observables $\mathcal{O}$ in terms of the classical color field we must perform averaging over the set of sources $\rho$, defined as

$$
\langle\mathcal{O}\rangle_{\rho} \equiv \int \mathcal{D} \rho W[\rho] \mathcal{O}[\rho],
$$

where the weight function $W[\rho]$ is the distribution of color sources. In this work, we use the simple MV model approximation in which $W[\rho]$ is obtained by considering a Gaussian distribution

$$
W[\rho(\mathbf{x})]=\exp \left[-\int d^{2} x \frac{\rho^{2}(\mathbf{x})}{2 \lambda}\right],
$$

where $\lambda$ describes the color source density of the nucleus and we assume that the color sources only depends on the transverse position $\mathbf{x}$.

First, let us consider the two-point correlation function

$$
S(\mathbf{x}-\mathbf{y}) \equiv \frac{1}{N^{2}-1}\left\langle\operatorname{Tr}\left(\mathcal{U}(\mathbf{x}) \mathcal{U}^{\dagger}(\mathbf{y})\right)\right\rangle_{\rho},
$$

where the adjoint Wilson line $\mathcal{U}(\mathbf{x})$ in the eikonal approximation simplifies to

$$
\mathcal{U}(\mathbf{x})=\exp \left[-i g T_{A}^{a} \Phi_{a}(\mathbf{x})\right]
$$

with $\Phi(\mathbf{x})=\frac{1}{\partial_{T}^{2}} \rho_{a}(\mathbf{x})$. Using the relation

$$
\frac{1}{\partial_{T}^{2}} \rho_{a}(\mathbf{x})=\int d^{2} z G(\mathbf{x}-\mathbf{z}) \rho_{a}(\mathbf{z})
$$

where $G$ is the Green's function associated with the twodimensional Laplacian and satisfies

$$
G(\mathbf{x}-\mathbf{z})=\int \frac{d^{2} k}{(2 \pi)^{2}} \frac{e^{i \mathbf{k} \cdot(\mathbf{x}-\mathbf{z})}}{k_{T}^{2}},
$$

allows us to rewrite the Wilson line in Eq. (B4) as

$$
\mathcal{U}(\mathbf{x})=\exp \left[-i g T_{A}^{a} \int d^{2} z G(\mathbf{x}-\mathbf{z}) \rho_{a}(\mathbf{z})\right] .
$$

Now the expectation value of two adjoint Wilson line in Eq. (B3) can be written as

$$
\begin{aligned}
\left\langle\mathcal{U}(\mathbf{x}) \mathcal{U}^{\dagger}(\mathbf{y})\right\rangle_{\rho}= & \int \mathcal{D} \rho \exp \left[-\int d^{2} z\left(\frac{1}{2 \lambda} \rho^{2}\right.\right. \\
& +i g(G(\mathbf{x}-\mathbf{z})-G(\mathbf{y}-\mathbf{z})) \rho)] .
\end{aligned}
$$

Performing the Gaussian integral leads to the result

$$
\begin{aligned}
\left\langle\mathcal{U}(\mathbf{x}) \mathcal{U}^{\dagger}(\mathbf{y})\right\rangle_{\rho}= & \exp \left[-\frac{\lambda g^{2}}{2}\left(T_{A}^{a} T_{A}^{a}\right) \int d^{2} z(G(\mathbf{x}-\mathbf{z})\right. \\
& \left.-G(\mathbf{y}-\mathbf{z}))^{2}\right]
\end{aligned}
$$

where the matrix $T_{A}^{a} T_{A}^{a}=C_{A} \mathbf{1}_{N^{2}-1}$ is the adjoint Casimir $C_{A}=N$ times an adjoint unit matrix $\mathbf{1}_{N^{2}-1}$. Exponentiation is trivial and we have

$$
\begin{aligned}
S(\mathbf{x}-\mathbf{y}) & =\frac{1}{N_{c}^{2}-1}\left\langle\operatorname{Tr}\left(\mathcal{U}(\mathbf{x}) \mathcal{U}^{\dagger}(\mathbf{y})\right)\right\rangle_{\rho} \\
& =\exp \left[-Q_{s}^{2} D(|\mathbf{x}-\mathbf{y}|)\right],
\end{aligned}
$$


where $Q_{s}^{2}=\frac{1}{2} \lambda g^{2} C_{A}$ and by using the integral representation of the Green's function we have defined the dipole function $D$ as

$$
\begin{aligned}
D(|\mathbf{x}-\mathbf{y}|) & \equiv \int d^{2} z(G(\mathbf{x}-\mathbf{z})-G(\mathbf{y}-\mathbf{z}))^{2} \\
& =\int \frac{d^{2} k}{(2 \pi)^{2}} \frac{2}{\left(k_{T}^{2}\right)^{2}}\left[1-e^{i \mathbf{k} \cdot(\mathbf{x}-\mathbf{y})}\right] .
\end{aligned}
$$

The remaining Fourier integral in Eq. (B11) is IR divergent. This can for example be regulated by replacing $k_{T}^{2}$ by $k_{T}^{2}+m_{\mathrm{IR}}^{2}$,

$$
\begin{aligned}
D_{m}(\mathbf{r}) & =\int \frac{d^{2} k}{(2 \pi)^{2}} \frac{2}{\left(k_{T}^{2}+m_{\mathrm{IR}}^{2}\right)^{2}}\left(1-e^{i \mathbf{k} \cdot \mathbf{r}}\right) \\
& =\frac{1}{2 \pi m_{\mathrm{IR}}^{2}}\left[1-m_{\mathrm{IR}} r_{T} K_{1}\left(m_{\mathrm{IR}} r_{T}\right)\right],
\end{aligned}
$$

where $\mathbf{r}=\mathbf{x}-\mathbf{y}$ and $r_{T}=|\mathbf{x}-\mathbf{y}|$.

Similarly, for the expectation value of a single adjoint Wilson line one obtains

$$
\begin{aligned}
\langle\mathcal{U}(\mathbf{x})\rangle_{\rho} & =\exp \left[-Q_{s}^{2} \mathbf{1}_{N^{2}-1} \int d^{2} z G^{2}(\mathbf{x}-\mathbf{z})\right] \\
& =\mathbf{1}_{N^{2}-1} \exp \left[-Q_{s}^{2} \int \frac{d^{2} x}{16 \pi^{2}} \log ^{2}\left(\frac{1}{x^{2} \Lambda^{2}}+1\right)\right] \\
& =\mathbf{1}_{N^{2}-1} \exp \left[-\frac{\pi Q_{s}^{2}}{48 \Lambda^{2}}\right]
\end{aligned}
$$

where we regulated IR and UV of the Green's function (B6) by

$$
G(\mathbf{x})=\frac{1}{4 \pi} \log \left(\frac{1}{x_{T}^{2} \Lambda^{2}}+1\right) .
$$

Comparing with the mass-regulated Green's function, $\Lambda=\frac{1}{2} m_{\mathrm{IR}} e^{\gamma_{E}}$. The expectation value of a single Wilson line thus effectively vanishes.

\section{Asymptotic behavior}

Let us then study the asymptotic behavior of Eq. (B12). Expanding in small $m_{\mathrm{IR}}^{2}$ gives

$$
\begin{aligned}
D_{m}\left(r_{T}\right) & =\frac{r_{T}^{2}}{4 \pi}\left(\log \left(\frac{2}{r_{T} m_{\mathrm{IR}}}\right)-\gamma_{E}+\frac{1}{2}\right)+\mathcal{O}\left(m_{\mathrm{IR}}^{4}\right) \\
& \equiv \frac{r_{T}^{2}}{4 \pi} \log \left(\frac{1}{r_{T} \Lambda_{\mathrm{QCD}}}\right),
\end{aligned}
$$

where $\Lambda_{\mathrm{QCD}}=\frac{1}{2} e^{\gamma_{E}-\frac{1}{2}} m_{\mathrm{IR}}=0.5401 m_{\mathrm{IR}}$. How the mass regularization operates is clearly seen from Fig. 7, where the function Eq. (B12) and its small $m_{\mathrm{IR}}$ approximation (B15) are plotted for $m_{\mathrm{IR}}=0.2$. The maximum of the small

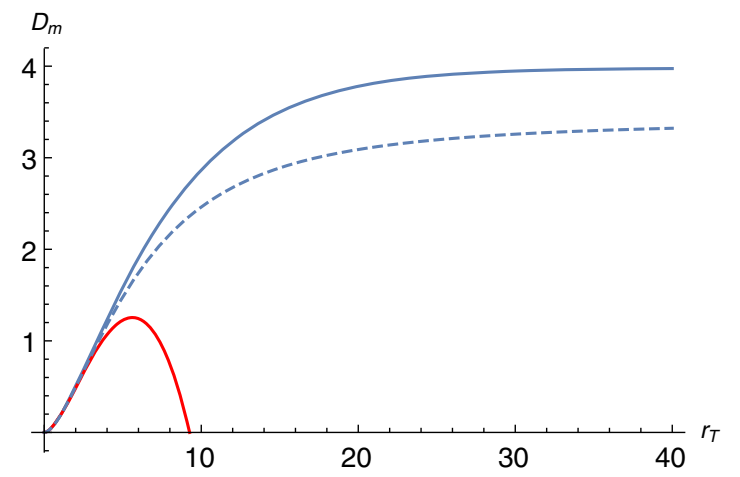

FIG. 7. The function $D_{m}\left(r_{T}\right)$ in Eq. (B12) and its small $m_{\mathrm{IR}}$ approximation in Eq. (B15) plotted for $m_{\mathrm{IR}}=0.2$. The maximum of the red curve is at $r_{T}=e^{-1 / 2} / \Lambda_{\mathrm{QCD}}$. The dashed curve is the approximation (B16).

$m_{\mathrm{IR}}$ approximation is at $r_{T}=2 e^{-\gamma_{E}} / m_{\mathrm{IR}}=1.123 / m_{\mathrm{IR}}$. Figure 7 also shows that another simple way of extending the small $r_{T}$ approximation (B15) to larger $r_{T}$ is by replacing it by

$$
D_{\Lambda}\left(r_{T}\right)=\frac{r_{T}^{2}}{8 \pi} \log \left(\frac{1}{\left(r_{T} \Lambda\right)^{2}}+1\right) .
$$

For the Fourier transform of correlation function $S\left(r_{T}\right)$ we have

$$
\begin{aligned}
S\left(q_{T}\right)= & \int d^{2} r e^{-i \mathbf{q} \cdot \mathbf{r}} S\left(r_{T}\right) \\
= & 2 \pi \int_{0}^{\infty} d r r J_{0}\left(q_{T} r\right) \exp \left[-Q^{2} D(r)\right] \\
= & \frac{2 \pi}{q_{T}^{2}} \int_{0}^{\infty} d r r J_{0}(r) \\
& \times \exp \left[-\frac{Q^{2}}{8 \pi q_{T}^{2}} r^{2} \log \left(\frac{q_{T}^{2}}{(0.10802 r)^{2}}+1\right)\right],
\end{aligned}
$$

where in the second form $\Lambda$ regularization Eq. (B16) with $\Lambda=0.5401 m_{\mathrm{IR}}=0.10802$ corresponding to $m_{\mathrm{IR}}=0.2$ has been used. Because of the Bessel function the integral is rapidly varying and special methods can be developed for accurate evaluation [33]. In Mathematica NIntegrate with Method $\rightarrow$ "Extrapolating Oscillatory" can be applied. At large $r$ the integrand is exponentially small, $\sim \exp \left(-Q^{2} /\left(2 \pi m_{\mathrm{IR}}^{2}\right)\right)$.

For $q_{T} \gg Q_{s}^{A}$, the correlation function $S\left(q_{T}\right)$ goes like [31]

$$
S\left(q_{T}\right)=\frac{2\left(Q_{s}^{A}\right)^{2}}{q_{T}^{4}}+\frac{8\left(Q_{s}^{A}\right)^{4}}{\pi q_{T}^{6}}\left(\log \frac{q_{T}}{\Lambda_{\mathrm{QCD}}}-1\right) .
$$

This can be derived by expanding the exponent in Eq. (B18) with mass regularization, 

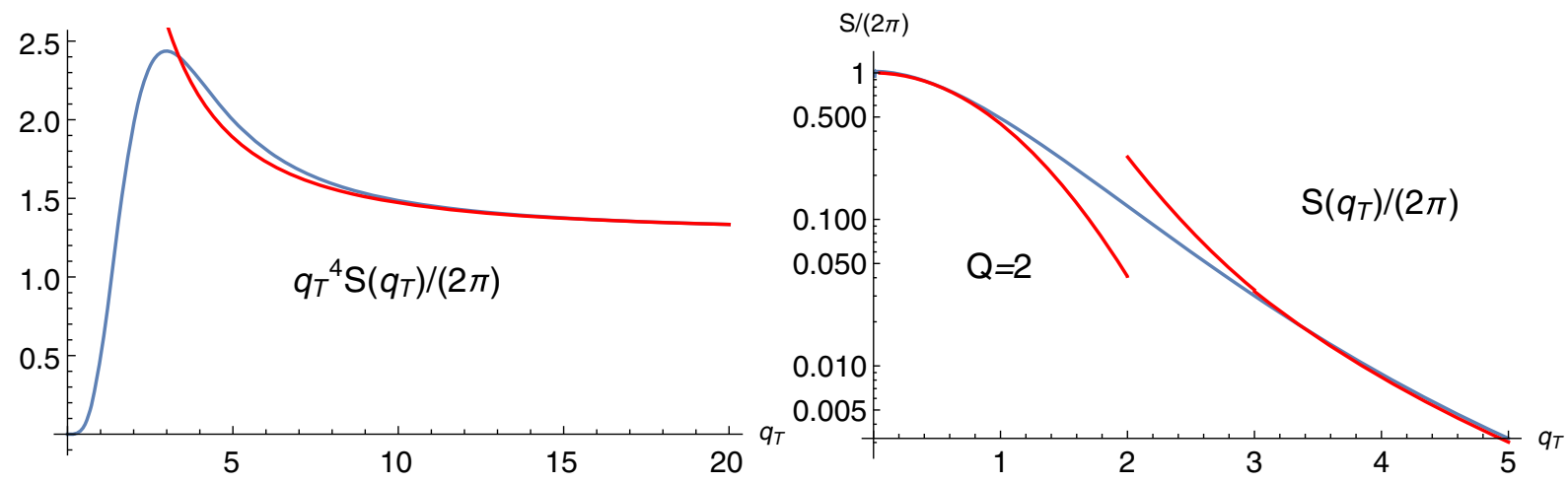

FIG. 8. The left panel shows the plot of $q_{T}^{4} S\left(q_{T}\right) /(2 \pi)$ for $Q_{s}^{A}=2, \Lambda=0.10802$ together with the large $q_{T}$ approximation (B19) with the logarithmic factor $\log \left(q_{T} / 0.468\right)$ (red). The right panel shows unscaled $S\left(q_{T}\right)$ together with a Gaussian $q_{T}<2$ approximation $1.025 \exp \left(-0.8 q_{T}^{2}\right)$ and the large $q_{T}>2$ approximation.

$$
\begin{aligned}
S\left(q_{T}\right)= & \int d^{2} r e^{-i \mathbf{q} \cdot \mathbf{r}}\left[1-\left(Q_{s}^{A}\right)^{2} \int \frac{d^{2} p}{(2 \pi)^{2}} \frac{2}{\left(p_{T}^{2}+m_{\mathrm{IR}}^{2}\right)^{2}}\left(1-e^{i \mathbf{p} \cdot \mathbf{r}}\right)\right. \\
& \left.+\frac{\left(Q_{s}^{A}\right)^{4}}{2} \int \frac{d^{2} p_{1}}{(2 \pi)^{2}} \frac{d^{2} p_{2}}{(2 \pi)^{2}} \frac{4\left(1-e^{i \mathbf{p}_{1} \cdot \mathbf{r}}-e^{i \mathbf{p}_{2} \cdot \mathbf{r}}+e^{i\left(\mathbf{p}_{1}+\mathbf{p}_{2}\right) \cdot \mathbf{r}}\right)}{\left(p_{1 T}^{2}+m_{\mathrm{IR}}^{2}\right)^{2}\left(p_{2 T}^{2}+m_{\mathrm{IR}}^{2}\right)^{2}}\right] \\
= & \frac{2\left(Q_{s}^{A}\right)^{2}}{q_{T}^{4}}+\frac{\left(Q_{s}^{A}\right)^{4}}{2}\left[-\frac{2}{\pi m_{\mathrm{IR}}^{2} q_{T}^{4}}+4 \int \frac{d^{2} p}{(2 \pi)^{2}} \frac{1}{\left(p^{2}+m_{\mathrm{IR}}^{2}\right)^{2}\left(|\mathbf{q}-\mathbf{p}|^{2}+m_{\mathrm{IR}}^{2}\right)^{2}}\right],
\end{aligned}
$$

where the $\mathbf{r}$ integral is carried out first and in the remaining p integral one has taken the limit $m_{\mathrm{IR}} \rightarrow 0$; the powerlike $1 / m_{\mathrm{IR}}^{2}$ divergence then cancels. This gives the result in Eq. (B19), where the scale of the log depends on the regularization method used.

Figure 8 (left panel) shows a plot of $q_{T}^{4} S\left(q_{T}\right) /(2 \pi)$ for $Q_{s}^{A}=2$ and $\Lambda=0.10802$ together with the approximation (B19) combined with the logarithmic factor $\log \left(q_{T} / 0.468\right)$. This scale of the log gives a very good fit in the large $q_{T}$ range but undershoots below. This is an effect of order $Q^{6} / q_{T}^{8}$. The right panel shows $S\left(q_{T}\right) /(2 \pi)$ without the scaling together with a small $q_{T}$ fit $1.025 \exp \left(-0.8 q_{T}^{2}\right)$ and the large $q_{T}$ approximation (B19). The transition region is at $q_{T} \approx Q_{s}^{A}=2$.

\section{APPENDIX C: AVERAGE OVER THE CLASSICAL COLOR SPACE}

Here we show how to calculate color average over the classical color charges in Eq. (42). After performing the square in Eq. (42), it is clear that there are four different color structures to be evaluated,

$$
\begin{aligned}
& \left\langle\mathcal{U}_{a d}(\mathbf{x}) \mathcal{U}_{d b}^{\dagger}(\mathbf{0}) Q^{b} Q^{c} \mathcal{U}_{c e}(\mathbf{0}) \mathcal{U}_{e a}^{\dagger}(\mathbf{y})\right\rangle_{Q}, \\
& \left\langle\mathcal{U}_{a d}(\mathbf{x}) \mathcal{U}_{d b}^{\dagger}(\mathbf{0}) Q^{b} Q^{a}\right\rangle_{Q}, \\
& \left\langle Q^{a} Q^{c} \mathcal{U}_{c e}(\mathbf{0}) \mathcal{U}_{e a}^{\dagger}(\mathbf{y})\right\rangle_{Q} \\
& \left\langle Q^{a} Q^{a}\right\rangle_{Q} .
\end{aligned}
$$

By using the relation given in Eq. (7) and the unitarity condition between two adjoint Wilson lines $\mathcal{U}(\mathbf{0}) \mathcal{U}^{\dagger}(\mathbf{0})=$ $\mathbf{1}_{N^{2}-1}$, we obtain the results

$$
\begin{aligned}
\left\langle\mathcal{U}_{a d}(\mathbf{x}) \mathcal{U}_{d b}^{\dagger}(\mathbf{0}) Q^{b} Q^{c} \mathcal{U}_{c e}(\mathbf{0}) \mathcal{U}_{e a}^{\dagger}(\mathbf{y})\right\rangle_{Q} & =\frac{1}{2} \operatorname{Tr}\left(\mathcal{U}(\mathbf{x}) \mathcal{U}^{\dagger}(\mathbf{y})\right), \\
\left\langle\mathcal{U}_{a d}(\mathbf{x}) \mathcal{U}_{d b}^{\dagger}(\mathbf{0}) Q^{b} Q^{a}\right\rangle_{Q} & =\frac{1}{2} \operatorname{Tr}\left(\mathcal{U}(\mathbf{x}) \mathcal{U}^{\dagger}(\mathbf{0})\right), \\
\left\langle Q^{a} Q^{c} \mathcal{U}_{c e}(\mathbf{0}) \mathcal{U}_{e a}^{\dagger}(\mathbf{y})\right\rangle_{Q} & =\frac{1}{2} \operatorname{Tr}\left(\mathcal{U}(\mathbf{y}) \mathcal{U}^{\dagger}(\mathbf{0})\right),
\end{aligned}
$$

and $\left\langle Q^{a} Q^{a}\right\rangle_{Q}=\frac{N^{2}-1}{2}$. Substituting these results into Eq. (42) gives the expression in Eq. (43). 
[1] K. Kajantie, L. D. McLerran, and R. Paatelainen, Gluon radiation from a classical point particle, Phys. Rev. D 100, 054011 (2019).

[2] L. D. McLerran and R. Venugopalan, Computing quark and gluon distribution functions for very large nuclei, Phys. Rev. D 49, 2233 (1994).

[3] L. D. McLerran and R. Venugopalan, Gluon distribution functions for very large nuclei at small transverse momentum, Phys. Rev. D 49, 3352 (1994).

[4] Y. V. Kovchegov and A. H. Mueller, Gluon production in current nucleus and nucleon-nucleus collisions in a quasiclassical approximation, Nucl. Phys. B529, 451 (1998).

[5] A. Dumitru and L. D. McLerran, How protons shatter colored glass, Nucl. Phys. A700, 492 (2002).

[6] A. Kovner, L. D. McLerran, and H. Weigert, Gluon production from nonAbelian Weizsacker-Williams fields in nucleus-nucleus collisions, Phys. Rev. D 52, 6231 (1995).

[7] A. Kovner, L. D. McLerran, and H. Weigert, Gluon production at high transverse momentum in the McLerranVenugopalan model of nuclear structure functions, Phys. Rev. D 52, 3809 (1995).

[8] A. Krasnitz, Y. Nara, and R. Venugopalan, Coherent Gluon Production in Very High-Energy Heavy Ion Collisions, Phys. Rev. Lett. 87, 192302 (2001).

[9] T. Lappi, Production of gluons in the classical field model for heavy ion collisions, Phys. Rev. C 67, 054903 (2003).

[10] J. P. Blaizot, F. Gelis, and R. Venugopalan, High-energy pA collisions in the color glass condensate approach. 1. Gluon production and the Cronin effect, Nucl. Phys. A743, 13 (2004).

[11] A. Dumitru, A. Hayashigaki, and J. Jalilian-Marian, The color glass condensate and hadron production in the forward region, Nucl. Phys. A765, 464 (2006).

[12] F. Gelis and Y. Mehtar-Tani, Gluon propagation inside a high-energy nucleus, Phys. Rev. D 73, 034019 (2006).

[13] F. Gelis, A. M. Stasto, and R. Venugopalan, Limiting fragmentation in hadron-hadron collisions at high energies, Eur. Phys. J. C 48, 489 (2006).

[14] E. Iancu, C. Marquet, and G. Soyez, Forward gluon production in hadron-hadron scattering with Pomeron loops, Nucl. Phys. A780, 52 (2006).

[15] C. Marquet, Forward inclusive dijet production and azimuthal correlations in $\mathrm{p}(\mathrm{A})$ collisions, Nucl. Phys. A796, 41 (2007).

[16] J. Casalderrey-Solana and E. Iancu, Interference effects in medium-induced gluon radiation, J. High Energy Phys. 08 (2011) 015.

[17] Y. Mehtar-Tani, C. A. Salgado, and K. Tywoniuk, The radiation pattern of a QCD antenna in a dilute medium, J. High Energy Phys. 04 (2012) 064.
[18] N. Armesto, H. Ma, M. Martinez, Y. Mehtar-Tani, and C. A. Salgado, Interference between initial and final state radiation in a QCD medium, Phys. Lett. B 717, 280 (2012).

[19] N. Armesto, H. Ma, M. Martinez, Y. Mehtar-Tani, and C. A. Salgado, Coherence phenomena between initial and final state radiation in a dense QCD medium, J. High Energy Phys. 12 (2013) 052.

[20] R. Anishetty, P. Koehler, and L. D. McLerran, Central collisions between heavy nuclei at extremely highenergies: The fragmentation region, Phys. Rev. D 22 (1980) 2793.

[21] K. Kajantie and L. D. McLerran, Energy densities, initial conditions and hydrodynamic equations for ultrarelativistic nucleus-nucleus collisions, Nucl. Phys. B214, 261 (1983).

[22] E. Iancu, A. Leonidov, and L. D. McLerran, Nonlinear gluon evolution in the color glass condensate. 1., Nucl. Phys. A692, 583 (2001).

[23] E. Ferreiro, E. Iancu, A. Leonidov, and L. McLerran, Nonlinear gluon evolution in the color glass condensate. 2., Nucl. Phys. A703, 489 (2002).

[24] L. D. McLerran, S. Schlichting, and S. Sen, Spacetime picture of baryon stopping in the color-glass condensate, Phys. Rev. D 99, 074009 (2019).

[25] G. S. Denicol, C. Gale, S. Jeon, A. Monnai, B. Schenke, and C. Shen, Net-baryon diffusion in fluid-dynamic simulations of relativistic heavy-ion collisions, Phys. Rev. C 98, 034916 (2018).

[26] S. K. Wong, Field and particle equations for the classical Yang-Mills field and particles with isotopic spin, Nuovo Cimento A 65, 689 (1970).

[27] S. Mrowczynski, B. Schenke, and M. Strickland, Color instabilities in the quarkgluon plasma, Phys. Rep. 682, 1 (2017).

[28] D. F. Litim and C. Manuel, Semiclassical transport theory for nonAbelian plasmas, Phys. Rep. 364, 451 (2002).

[29] L. D. McLerran and R. Venugopalan, Gluon distribution functions for very large nuclei at small transverse momentum, Phys. Rev. D 49, 3352 (1994).

[30] J. F. Gunion and G. Bertsch, Hadronization by color bremsstrahlung, Phys. Rev. D 25, 746 (1982).

[31] F. Gelis and A. Peshier, Probing colored glass via $q$ anti- $q$ photoproduction, Nucl. Phys. A697, 879 (2002).

[32] F. Gelis and N. Tanji, Statistical fluctuations of correlators in the color glass condensate, arXiv:1811.08711.

[33] R. Piessens and M. Branders, Approximation for Bessel functions and their application in the computation of Hankel transforms, Comput. Math. Appl. 8, 305 (1982). 